\title{
Linc00261 inhibits metastasis and the WNT signaling pathway of pancreatic cancer by regulating a miR-552-5p/FOXO3 axis
}

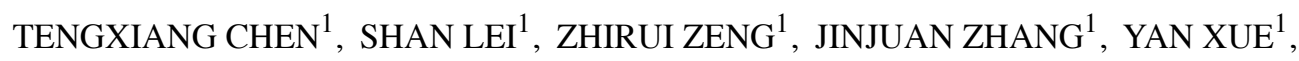

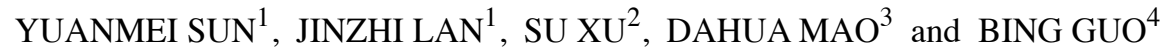 \\ ${ }^{1}$ Guizhou Provincial Key Laboratory of Pathogenesis and Drug Research on Common Chronic Diseases, \\ Department of Physiology, School of Basic Medical Sciences, Guizhou Medical University; \\ ${ }^{2}$ Department of Pathology, Affiliated Hospital of Guizhou Medical University; ${ }^{3}$ Department of Breast Surgery, \\ Wudang Affiliated Hospital, School of Clinical Medical Science; ${ }^{4}$ Guizhou Provincial Key Laboratory of \\ Pathogenesis and Drug Research on Common Chronic Diseases, Department of Pathophysiology, \\ School of Basic Medical Sciences, Guizhou Medical University, Guiyang, Guizhou 550009, P.R. China
}

Received July 28, 2019; Accepted January 9, 2020

DOI: $10.3892 /$ or.2020.7480

\begin{abstract}
The biological function of long non-coding RNA00261 (Linc00261) has been widely investigated in various types of cancer. The aim of the present study was to explore the role of Linc00261 in pancreatic cancer (PC). The expression of Linc00261 in patients with PC and PC cell lines was assessed using reverse transcription-quantitative PCR and the association of Linc00261 expression with survival was analyzed in the online database, GEPIA. The effects of Linc00261 on PC cell metastasis in vitro and in vivo were determined using a wound healing assay, Transwell invasion assays and a nude mouse model of liver metastasis. The relationship between Linc00261, the miR-552-5p/forkhead box $\mathrm{O} 3$ (FOXO3) axis and the Wnt signaling pathway were determined using bioinformatics analysis, dual luciferase assay and western blotting. Linc00261 expression was significantly decreased in PC tissues and cell lines, and reduced expression was associated with less favorable outcomes in patients with PC. Linc00261 overexpression inhibited migration and invasion of PC cells in vitro, whereas knockdown of Linc00261 increased migration and invasion. Linc00261 overexpression also decreased metastasis of PC cells in vivo. Linc00261 was revealed to directly bind to microRNA (miR)-552-5p and to decrease the expression of miR-552-5p. In addition, Linc00261 overexpression increased the expression
\end{abstract}

Correspondence to: Professor Tengxiang Chen, Guizhou Provincial Key Laboratory of Pathogenesis and Drug Research on Common Chronic Diseases, Department of Physiology, School of Basic Medical Sciences, Guizhou Medical University, Guiyang, Guizhou 550009, P.R. China

E-mail:txch@gmc.edu.cn

Key words: pancreatic cancer, metastasis, Linc00261, microRNA-552-5p, forkhead box O3 of FOXO3, a target gene of miR-552-5p, as well as inhibited the Wnt signaling pathway. Overexpression of miR-552-5p in Linc00261-overexpressing PC cells increased migration and invasion, as well as decreased the expression of FOXO3 and members of the Wnt signaling pathway. Collectively, the present study demonstrated that Linc00261 inhibited metastasis and the Wnt signaling pathway of PC by regulating the miR-552-5p/FOXO3 axis. Linc00261 may suppress the development of PC, and serve as a potential biomarker and effective target for the diagnosis and treatment of PC.

\section{Introduction}

Pancreatic cancer (PC) is the fourth most aggressive digestive cancer, and the 5-year overall survival rate is $<5 \%$ (1). Although therapeutic strategies for treatment of PC have improved, patient outcomes remain unsatisfactory due to a high degree of metastasis $(2,3)$. Epithelial-mesenchymal transition (EMT) is a key part of the metastatic process in PC. EMT is associated with significant changes in the expression of thousands of genes and is characterized by the downregulation of epithelial markers, such as E-cadherin, and the upregulation of mesenchymal markers, such as $\mathrm{N}$-cadherin and vimentin (4). The process of EMT is regulated by several signaling pathways, such as the MEK/ERK (5), AKT (6) and Wnt signaling pathways (7). Activation of the Wnt signaling pathway results in nuclear translocation of $\beta$-catenin, where it co-operates with TCF4 and increases the transcription of mesenchymal markers (8). Therefore, identification of upstream regulatory mechanisms of the Wnt signaling pathway and thus EMT may serve as targets for treatment of patients with PC.

Epigenetic regulation, including through non-coding RNAs, are upstream regulatory mechanisms which may regulate the Wnt signaling pathway and thus EMT (9). Long noncoding RNAs (lncRNAs) are a type of non-coding RNA $>200$ nucleotides in length. Competing endogenous RNAs (ceRNAs) sponge target microRNAs (miRNAs) by binding to the $3^{\prime}$ untranslated regions (3'UTR) of the target miRNAs 
and mRNAs and regulate their expression (10). Various lncRNAs have been demonstrated to be involved in the metastasis of PC (11). Yang et al (12) demonstrated that lncRNA DLX6-AS1 expression was increased in PC tissues and cell lines, and modulated the $\mathrm{Wnt} / \beta$-catenin pathway to promote proliferation, migration and invasion of cells by sponging miR-497-5p (12). LincRNA H19 activated the Wnt signaling pathway and promoted the metastasis of PC cells by regulating the miR-194/PFTK1 axis (13). The biological functions and associated mechanisms of Linc00261 have been demonstrated in various types of cancer $(14,15)$. Linc00261 expression was reduced in non-small cell lung cancer cells and had the capacity to inhibit the progression of non-small cell lung cancer (16). Linc00261 suppressed proliferation and migration of colon cancer by sponging miR-324-3p (17). However, the role of Linc00261 in PC is unknown, to the best of our knowledge.

Forkhead box $\mathrm{O} 3$ (FOXO3) is member of the forkhead box $\mathrm{O}$ transcription factors, which is involved in EMT by regulating the Wnt signaling pathway (18). Recently, numerous studies have revealed that FOXO3 is inactivated in different types of cancer and may serve as a tumor suppressor (19). In PC, by inhibiting the Wnt signaling pathway and thus EMT, FOXO3 also exhibited an inhibitory effect on metastasis in vitro and in vivo (20). However, the molecular regulatory mechanism by which FOXO3 results in these effects are not completely understood.

In the present study, the effect of Linc00261 on the metastasis of PC cells, and the association between Linc00261, EMT and metastasis was assessed. Linc00261 overexpression inhibited metastasis of PC cells, EMT and the Wnt signaling pathway by regulating the miR-552-5p/FOXO3 axis. Linc00261 may serve as a suppressive lncRNA in PC and thus may be a potentially useful biomarker for the diagnosis of PC and effective target for treatment.

\section{Materials and methods}

Clinical specimens. A total of 54 pairs of PC tissues and corresponding adjacent non-tumor tissues were collected at the Affiliated Hospital of Guizhou Medical University (Guizhou, China) between July 2014 and March 2019. The mean age of patients enrolled in the present was $55 \pm 7.4$ years, (range, 45-71 years) with 25 males and 29 females. None of patients enrolled in the present study received neoadjuvant chemotherapy, radiotherapy or immunotherapy prior to surgery. The present study was approved by the Ethics Committee of Guizhou Medical University and performed in accordance with the Declaration of Helsinki (Approval no. 2019LS146). All patients provided written informed consent for participation.

Bioinformatics method. The expression of Linc00261 in PC tissues and adjacent tissues was first assessed by online database GEPIA (URL: http://gepia.cancer-pku.cn/). LogFC $>1$ and a P-value $<0.05$ was set as cut-offs. TargetScan (version 7.2; URL: http://www.targetscan.org/vert_72/) was used to determine the target miRNA of Linc00261, while the target gene of target miRNA was determined by TargetScan and miRwalk (version 3.0; URL: http://mirwalk.umm.uni-heidelberg.de/). The pathways that the target genes were enriched in, were analysed using R software (version: 3.5.2; The R Foundation;
URL: https://www.r-project.org/). $\mathrm{P}<0.05$ was used as a threshold for a pathway to be considered significantly enriched.

Cell culture and transfection. A total of 6 PC cell lines, CFPAC-1 (liver metastasis derivation, metastasis potential), AsPC-1 (ascites derivation, metastasis potential), MIA-PaCa-2 (primary tumor, non-metastasis potential), Capan-2 (primary tumor, non-metastasis potential), BXPC-3 (primary tumor, non-metastasis potential) and PANC-1 (primary tumor, metastasis potential), and the normal pancreatic duct epithelial cell line HPDE were all purchased from American Type Culture Collection. CFPAC-1, MIA-PaCa-2 and PANC-1 cells were cultured in high-glucose DMEM medium (Gibco; Thermo Fisher Scientific, Inc.), whereas CFPAC-1, AsPC-1, Capan-2 and BXPC-3 cells were cultured in RPMI-1640 medium (Gibco; Thermo Fisher Scientific, Inc.). All cell lines were cultured at $37^{\circ} \mathrm{C}$ in a humidified atmosphere containing $5 \% \mathrm{CO}_{2}$.

A Linc00261 expression lentivirus was generated by subcloning the PCR-amplified full-length human Linc00261 cDNA into the pMSCV retrovirus plasmid (GeneCopoeia, Inc.). Empty pMSCV retrovirus plasmid was used as a negative control for Linc00261 expression lentivirus. Linc00261-targeting and scramble short hairpin RNA (shRNA) oligonucleotides were cloned into the pSuper-retro-puro vector to generate pSuper-retro-Linc00261-RNAi. The shRNA sequences were: shLinc00261, CAGTCGCTTGGTTTGAGC TCAAATA; scramble, UUCUCCGAACGUGUCACGUTT. The miR-552-5p mimic and inhibitor were obtained from GeneCopoeia, Inc. PANC-1 and Mia-PaCa2 cells were seeded into 6 -well plates at a density of $1 \times 10^{5} /$ well. After the cells had adhered, a lentivirus was added according to manufacturer's protocol (MOI=5 for PANC-1, MOI=10 for MIA-PaCa2). To obtain stably overexpressing Linc00261 cells or stable knockdown cells, cells were selected for 14 days using $1 \mu \mathrm{g} / \mathrm{ml}$ puromycin $48 \mathrm{~h}$ after transfection.

Reverse transcription-quantitative $(R T-q) P C R$. Total RNA of tissues and cells were extracted using TRIzol reagent (Wuhan Boster Biological Technology, Ltd.). Total RNA was reverse-transcribed into cDNA using PrimeScript RT reagent kit (Yeasen Biotechnology, Co., Ltd.). qPCR was performed using SYBR Green Master mix (Yeasen, Biotechnology, Co., Ltd.). Relative expression was normalized to GAPDH and fold-change was calculated using the $2^{-\Delta \Delta \mathrm{Cq}}$ method (21). The sequences of the primers used were: Linc00261 forward, GTC AGAAGGAAAGGCCGTGA and reverse, TGAGCCGAG ATGAACAGGTG; miR-552-5p forward, CCGCACAGG TGACTG GTTAGA and reverse, GTGCAGGGTCCGAGGT; FOXO3 forward, CGGACAAACGGCTCACTCT and reverse, GGACCCGCATGAATCGACTAT; E-cadherin forward, CGAGAGCTACACGTTCACGG and reverse, GGGTGT CGAGGGAAAAATAGG; N-cadherin forward, GGGTGT CGAGGGAAAAATAGG and reverse, ATGCACATCCTT CGATAAGACTG; vimentin forward, GACGCCATCAAC ACCGAGTT and reverse, CTTTGTCGTTGGTTAGCTGGT; and GAPDH forward, AGAAGGCTGGGGCTCATTTG and reverse, AGGGGCCATCCACAGTCTTC.

Wound healing assay. Total $5 \times 10^{5} \mathrm{PC}$ cells were seeded into 6 -well plates and cultured until they reached a confluence of 
Table I. The relationship between the expression of Linc00261 and various clinicopathological characteristics.

\begin{tabular}{|c|c|c|c|c|c|}
\hline Features & $n$ & Low & High & $\chi^{2}$ & P-value \\
\hline All cases & & 54 & 27 & 27 & \\
\hline Age & & & & 1.187 & 0.276 \\
\hline$<60$ & 28 & 12 & 16 & & \\
\hline$\geq 60$ & 26 & 15 & 11 & & \\
\hline Sex & & & & 1.2 & 0.273 \\
\hline Man & 30 & 13 & 17 & & \\
\hline Female & 24 & 14 & 10 & & \\
\hline Tumor size $(\mathrm{cm})$ & & & & 6.135 & 0.013 \\
\hline$<2$ & 23 & 7 & 16 & & \\
\hline$\geq 2$ & 31 & 20 & 11 & & \\
\hline Lymph node metastasis & & & & 9.012 & 0.003 \\
\hline Negative & 25 & 7 & 18 & & \\
\hline Positive & 29 & 20 & 9 & & \\
\hline TNM stage & & & & 14.7 & 0.001 \\
\hline I and II & 24 & 5 & 19 & & \\
\hline III and IV & 30 & 22 & 8 & & \\
\hline Distant metastasis & & & & 9.012 & 0.003 \\
\hline Negative & 26 & 7 & 18 & & \\
\hline Positive & 29 & 20 & 9 & & \\
\hline Perineural invasion & & & & 6.135 & 0.013 \\
\hline Negative & 23 & 7 & 16 & & \\
\hline Positive & 31 & 20 & 11 & & \\
\hline Blood vessel invasion & & & & 7.67 & 0.006 \\
\hline Negative & 22 & 6 & 16 & & \\
\hline Positive & 32 & 21 & 11 & & \\
\hline
\end{tabular}

90\%. A $200-\mu 1$ pipette tip was used to scratch the monolayer to create a wound. After scratching, the cells were washed using PBS to remove detached cells. Cells were subsequently incubated in the serum-free DMEM and cultured for $48 \mathrm{~h}$. Phase-contrast microscopy was used to capture the images in 6 random fields.

Transwell invasion assay. Cells were suspended in medium without FBS and a total of $1 \times 10^{5} \mathrm{PC}$ cells were then added to the upper chamber pre-coated with Matrigel (BD Biosciences), and DMEM supplemented with $20 \%$ FBS was added to the lower chamber. Cells were cultured at $37^{\circ} \mathrm{C}$ for $48 \mathrm{~h}$, after which, the cells which had invaded were fixed using $4 \%$ paraformaldehyde and stained using $0.5 \%$ crystal violet (Wuhan Boster Biological Technology, Ltd.) at room temperature for $30 \mathrm{~min}$. After washing with PBS, the chambers were air-dried and observed under an inverted light microscope (Olympus Corporation). The number of invaded cells in five random fields were counted using Image Pro-Plus (version: 6.0; Sevice and technology, URL: https://www.xrayscan. com/software-image-pro-plus/).

Animal model of liver metastasis. The nude mice used in the present study were obtained from Huafukang Biotechnology Co., Ltd. Animals were kept in specific pathogen-free condi- tions. NC and Linc00261 overexpression of PANC-1 cells ( $2 \times 10^{6}$ cells) were injected into the spleen of nude mice $(n=6$ per group). After intravenous anesthesia with pentobarbital at a concentration of $2.5 \mathrm{mg} / 100 \mathrm{~g}$, the mice were sacrificed via cervival dislocation in 10 weeks after injection, and the liver was harvested, weighed, imaged and embedded in $10 \%$ paraffin. All animal experiments were approved by the Ethics Committee of Guizhou Medical University (Approval no. 1900069).

$H \& E$ staining. The liver tissues were fixed in $4 \%$ paraformaldehyde for $30 \mathrm{~min}$ at room temperature and sectioned into $4 \mu \mathrm{m}$-thick sections. After heating to $60^{\circ} \mathrm{C}$ for $1 \mathrm{~h}$, the specimens were deparaffinized using xylene at room temperature and rehydrated in a descending graded series of ethanol $(100,80,60$ and $40 \%)$. Subsequently, the samples were stained with hematoxylin for $5 \mathrm{~min}$ and eosin for $10 \mathrm{~min}$ both at room temperature. After washing with PBS, images were captured using an upright metallurgical microscope (x200; Olympus Corp.).

Luciferase reporter assay. PC cells were plated in 6-well plates at density of $5 \times 10^{5}$. Subsequently, the cells were co-transfected with psiCHECK ${ }^{\mathrm{TM}}-2$ vector and miR-552-5p mimics. Then, the cells were lysed and used to measure firefly 
A
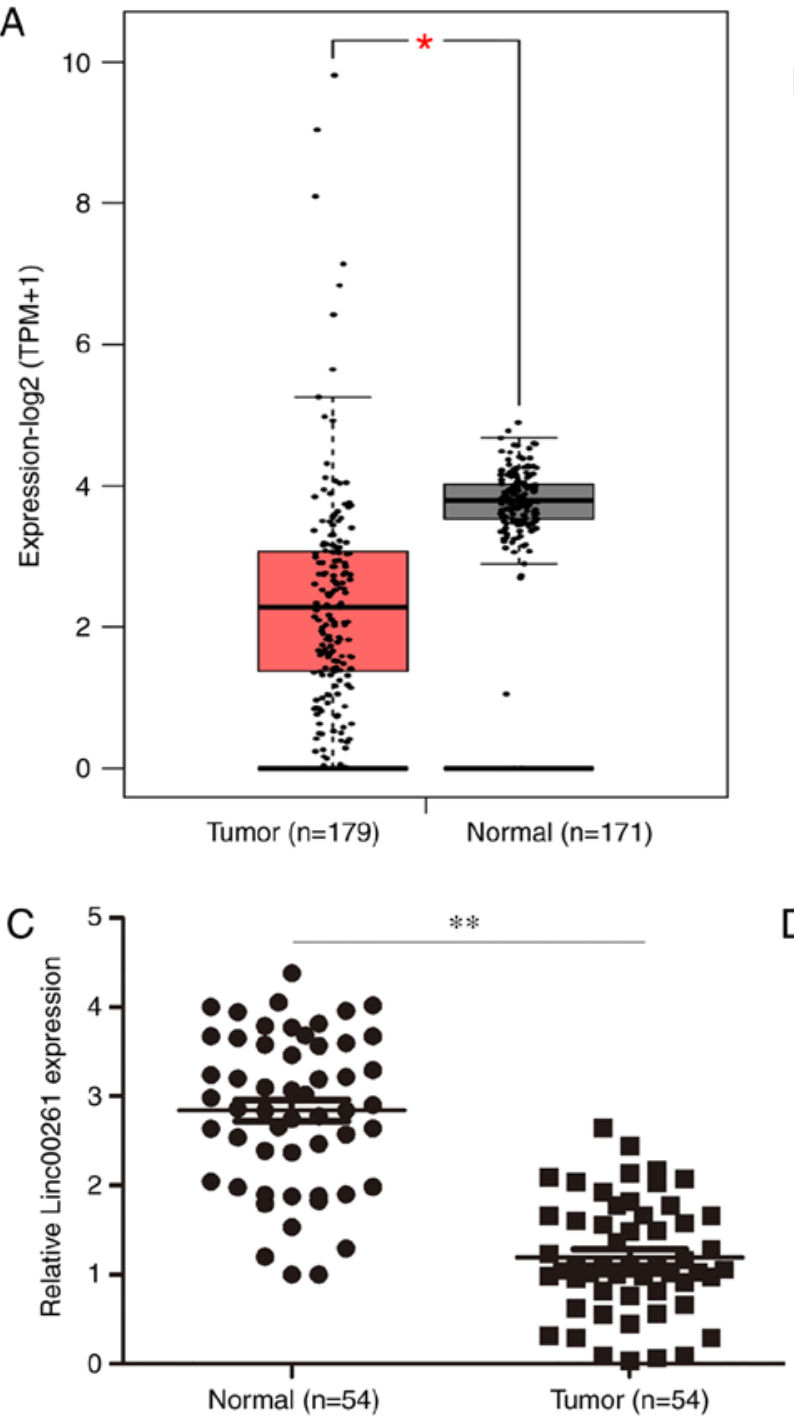

B

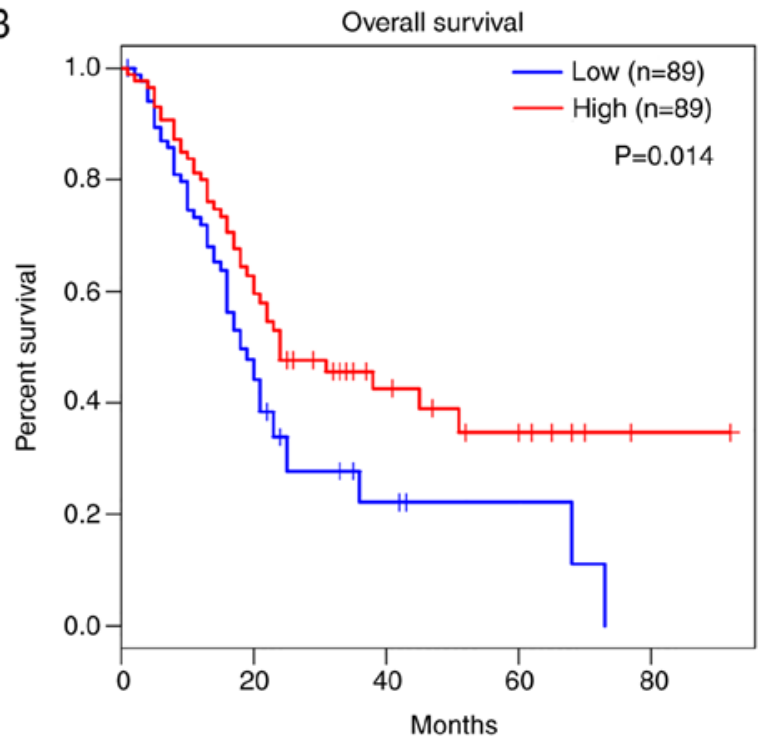

D

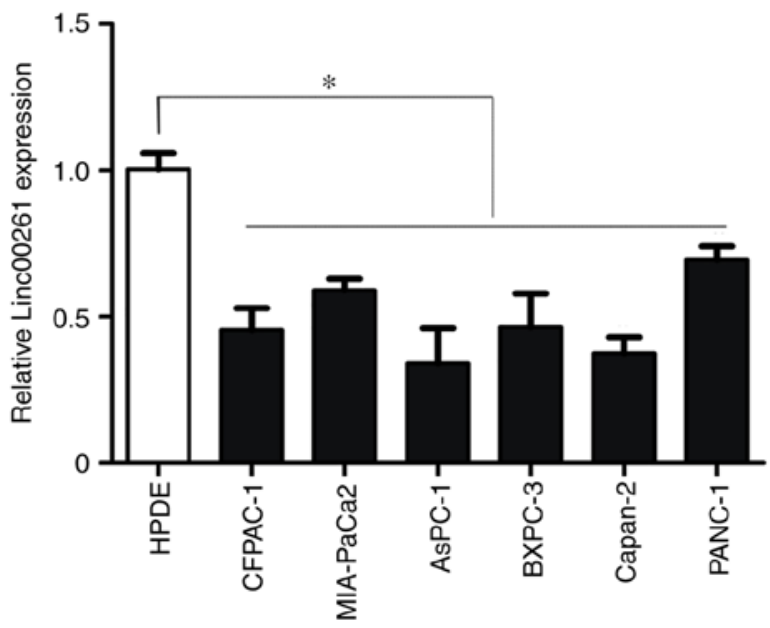

Figure 1. Linc00261 is significantly downregulated in PC. (A) The expression of Linc00261 in PC tissues and adjacent healthy tissues was analyzed using the online database, GEPIA. (B) Association between the prognosis and the expression of Linc00261 was analyzed using the online database, GEPIA. (C) Expression of Linc00261 was measured in 54 PC tissues and 54 adjacent non-tumor tissues using reverse transcription-quantitative PCR. (D) The expression of Linc00261 in the PC cell lines, CFPAC-1, AsPC-1, MIA-PaCa2, Capan-2, BXPC-3 and PANC-1 was significantly decreased compared with the HPDE cells. ${ }^{*} \mathrm{P}<0.05,{ }^{* *} \mathrm{P}<0.01$. PC, pancreatic cancer; HPDE, human pancreatic epithelium cells.

and Renilla luciferase activity according to manufacturer's protocol (Guangzhou RiboBio Co., Ltd.). Subsequently, the luciferase activity was normalized to the firefly luciferase internal control.

Western blotting. Total protein was extracted from PC cells using RIPA lysis buffer (Wuhan Boster Biological Technology, Ltd.), and the protein concentration was quantified using a bicinchoninic acid protein assay kit (Wuhan Boster Biological Technology, Ltd.). Proteins (30 $\mu \mathrm{g}$ per lane) were loaded on a $10 \%$ gel and resolved using SDS-PAGE and transferred to PVDF membranes (EMD Millipore). Membranes were blocked using 5\% skimmed milk, and subsequently incubated overnight using primary antibodies against target proteins, FOXO3 (cat. no. 10849-1-AP), $\beta$-catenin (cat. no. 51067-2-AP), TCF4 (cat. no. 22337-1-AP), E-cadherin (cat. no. 20874-1-AP), N-cadherin (cat. no. 22018-1-AP), vimentin (cat. no. 10366-1-AP) and GAPDH (cat. no. 60004-1-Ig) (all 1:1,000; ProteinTech Group, Inc.) at $4^{\circ} \mathrm{C}$. Subsequently, the membranes were washed using TBS-Tween twice, and subsequently the membranes were incubated with horseradish peroxidase-conjugated anti-mouse (cat. no. BA1051) and anti-rabbit (cat. no. BA1055) secondary antibody (all 1:2,500; Wuhan Boster Biological Technology, Ltd.) and signals were visualized using enhanced chemiluminescent reagent (Wuhan Boster Biological Technology, Ltd.). Image Pro-Plus software was used to analyze the expression of protein, while GAPDH was used as a loading control.

Statistical analysis. Statistical analysis was performed using SPSS version 21.0 (IBM Corp). Survival curves were plotted 
A
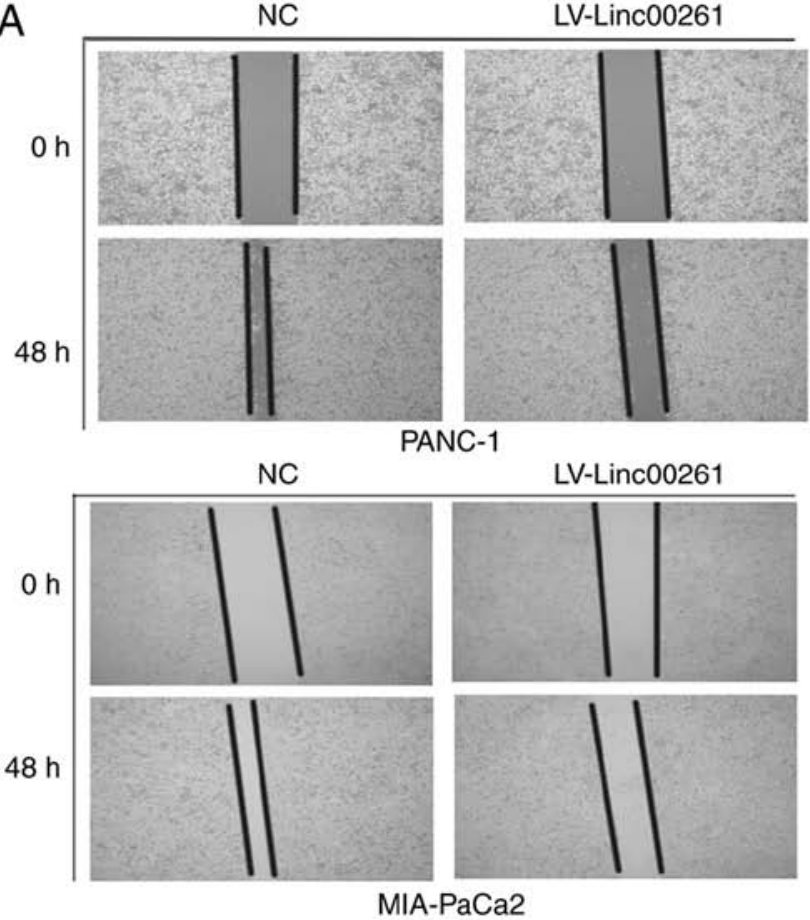

B

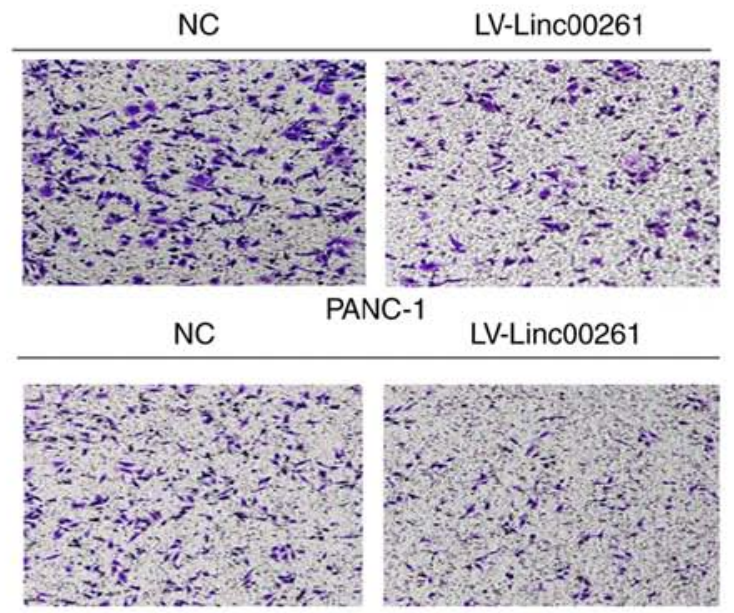

MIA-PaCa2

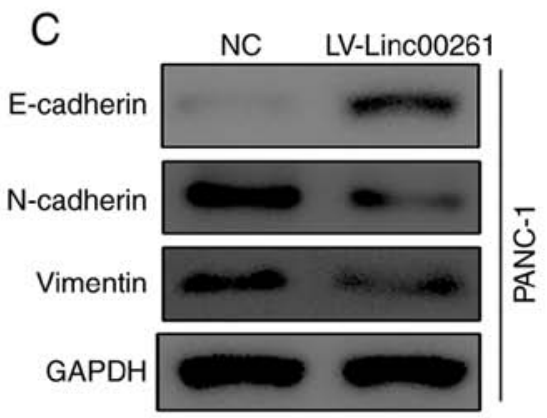

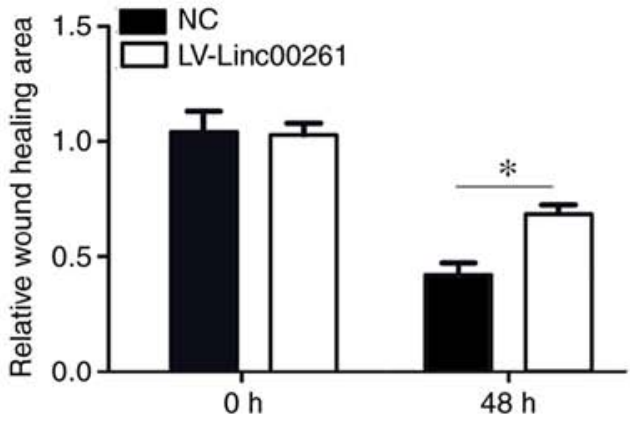
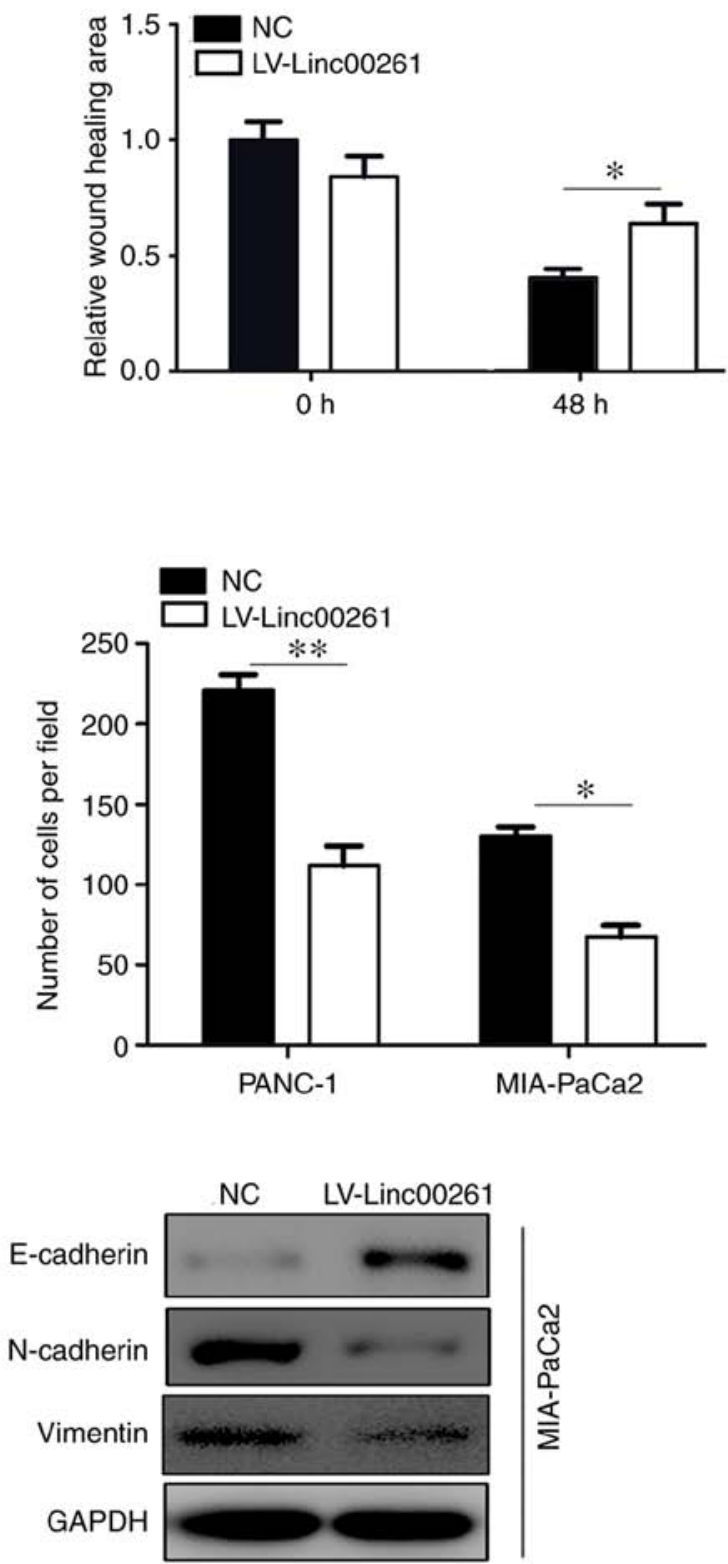

Figure 2. Linc00261 overexpression inhibits the migration and invasion of PC cells in vitro. PANC-1 and MIA-PaCa2 were transfected with empty vector or Linc00261 overexpression lentivirus. (A) A wound healing assay was used to assess the effect of Linc00261 overexpression on the migration of PC cells. (B) A Transwell invasion assay was used to detect the effect of Linc00261 overexpression on the invasion of PC cells. (C) Western blotting was used to detect the expression of three epithelial-mesenchymal transition markers, E-cadherin, $\mathrm{N}$-cadherin and vimentin, in Linc00261-overexpressing cells. ${ }^{*} \mathrm{P}<0.05,{ }^{*} \mathrm{P}<0.01$. PC, pancreatic cancer; LV-Linc00261, Linc00261-overexpressing cells; NC, negative control.

using Kaplan-Meier plotter (kmplot.com). Comparisons were performed using a one-way ANOVA combined with LSD-t test or a paired Student's t-test. $\mathrm{P}<0.05$ was considered to indicate a statistically significant difference.

\section{Results}

Linc00261 expression is decreased in PC. The expression and association of Linc00261 with clinical traits were exam- 
A

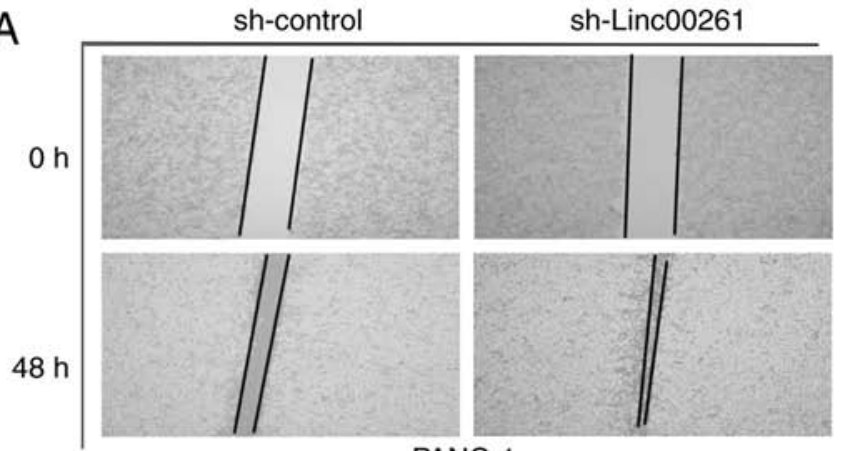

PANC-1

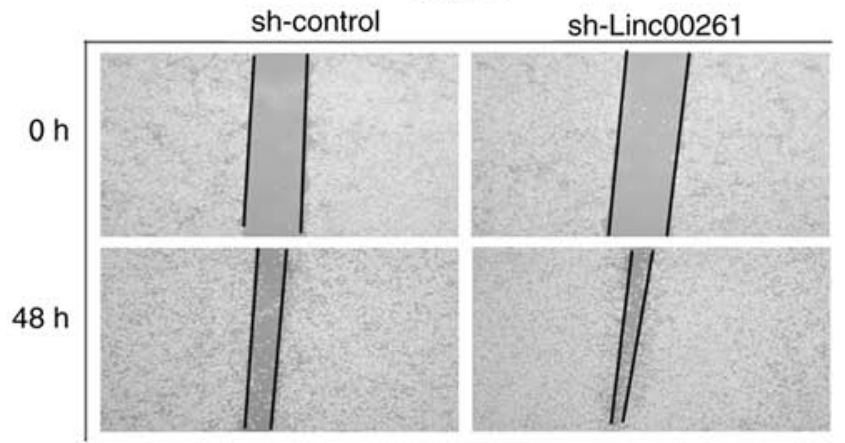

MIA-PaCa2

B

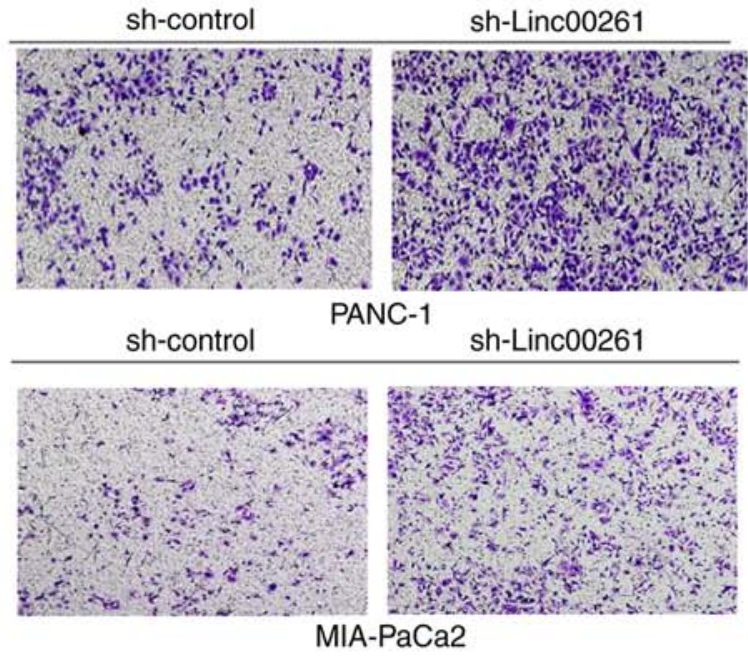

C

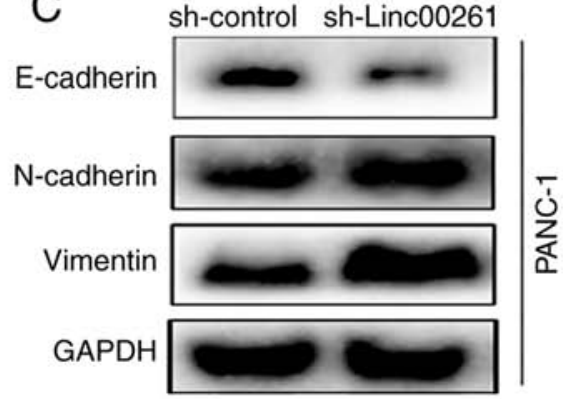

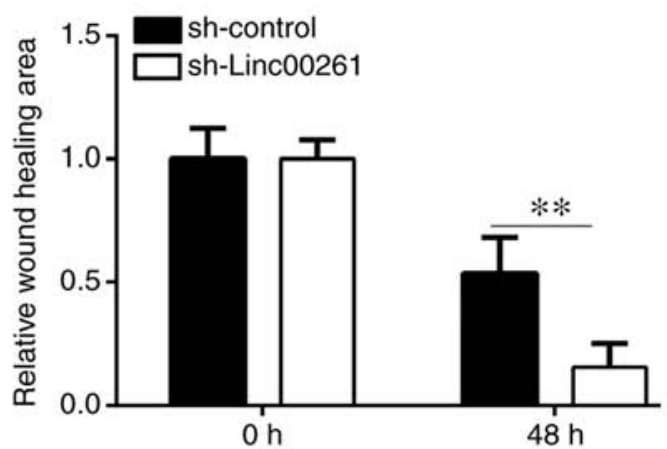
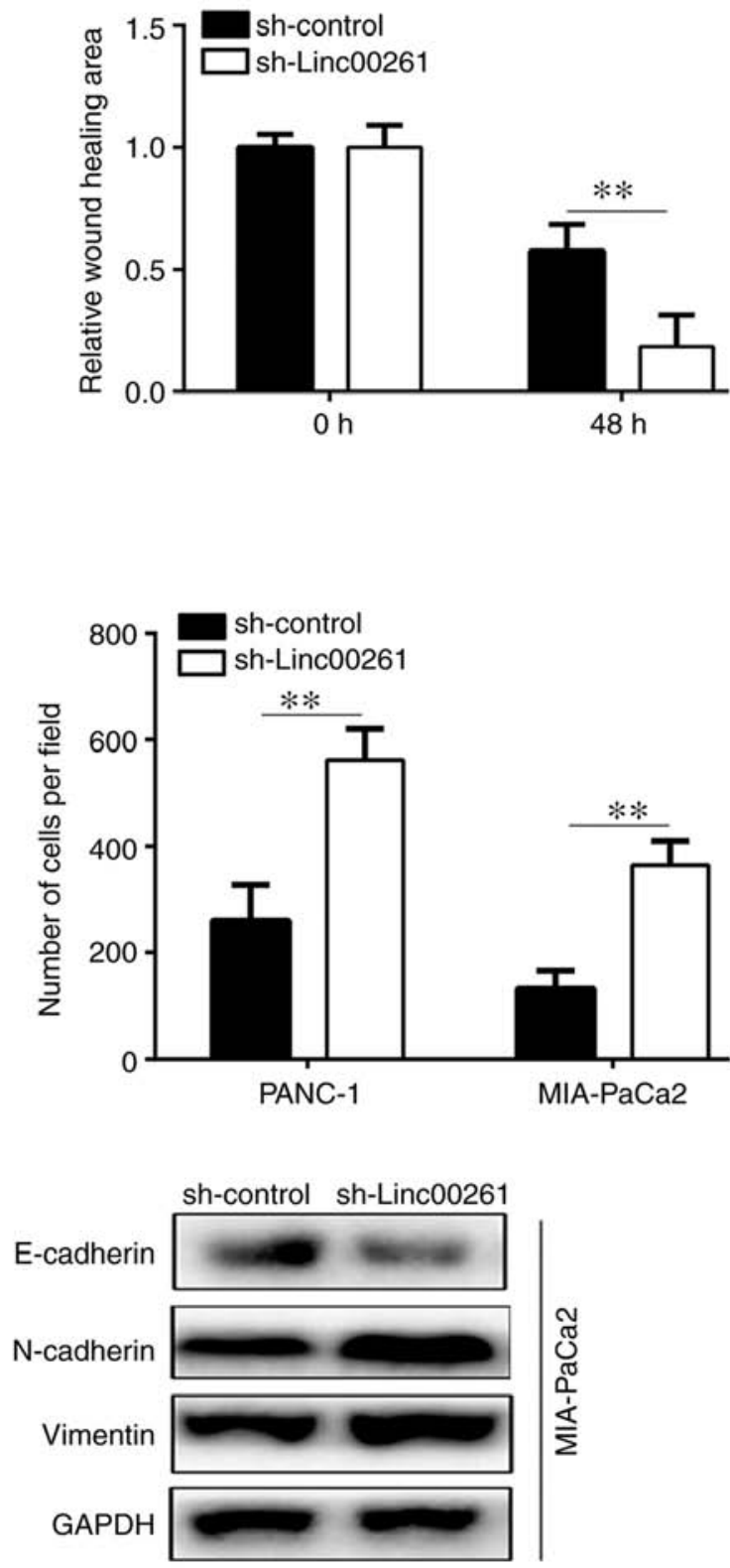

Figure 3. Linc00261 knockdown increases the migration and invasion of PC cells in vitro. PANC-1 and MIA-PaCa2 cells were transfected with scrambled shRNA and targeted Linc00261 shRNA, and used as the NC group and Linc00261-knockdown group, respectively. (A) A wound healing assay was used to assess the effects of Linc00261 knockdown on the migration of PC cells. (B) A Transwell invasion assay was used to assess the effects of Linc00261 knockdown on the invasion of PC cells. (C) Western blotting was used to detect the expression of three epithelial-mesenchymal transition markers, E-cadherin, $\mathrm{N}$-cadherin and vimentin in Linc00261-knockdown cells. ${ }^{* *} \mathrm{P}<0.01$. PC, pancreatic cancer; sh, short hairpin; NC, negative control represented by sh-Control; sh-Linc00261, Linc00261 knockdown.

ined. Bioinformatics analysis of data obtained from GEPIA revealed that Linc00261 was significantly reduced in PC tissues compared with the matching adjacent tissues (Fig. 1A; $\mathrm{P}<0.05)$. Reduced expression of Linc00261 in patients with 
A
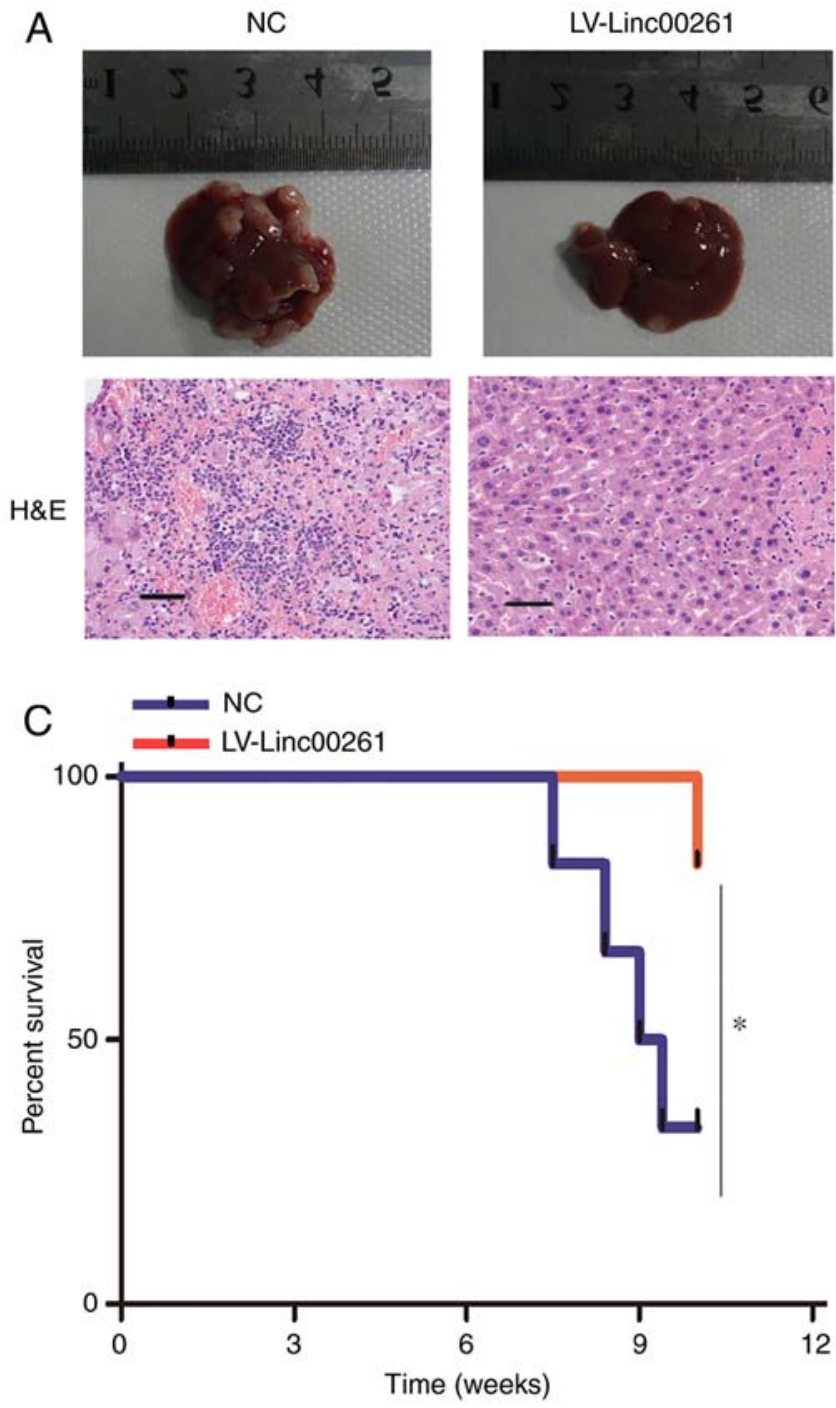

B

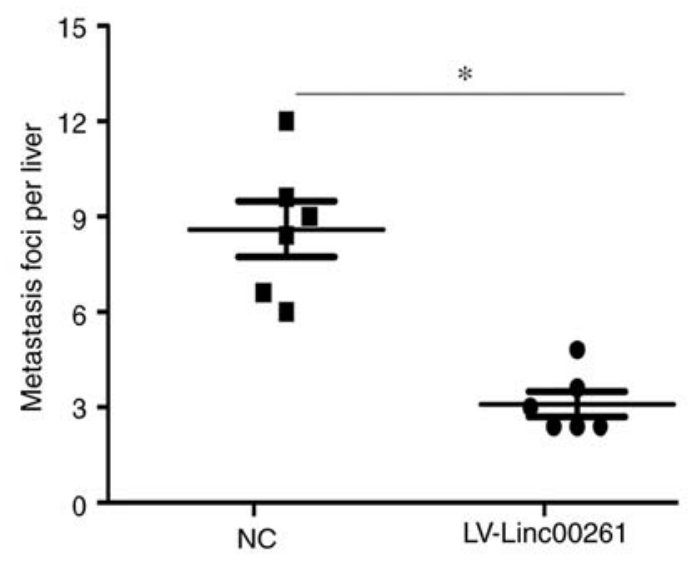

D

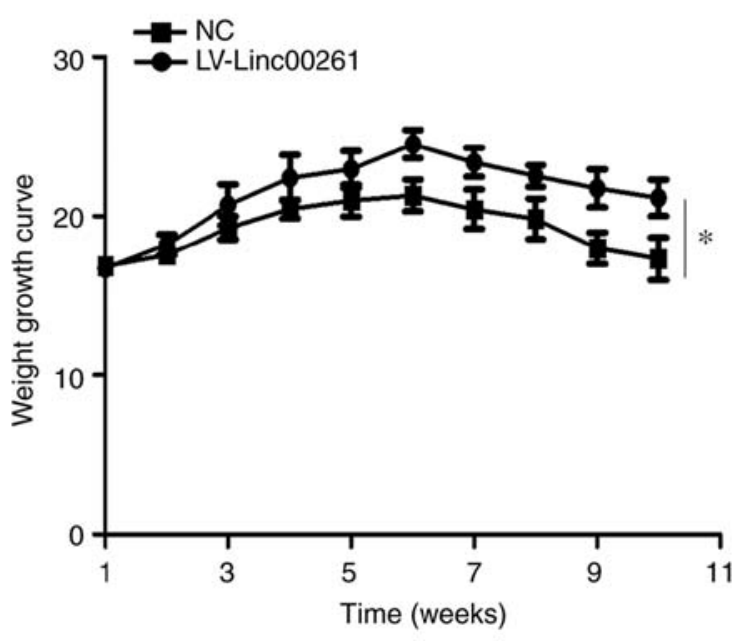

Figure 4. Linc00261 overexpression reduces metastasis of PC cells in vivo. (A) Images of the liver metastasis model. (B) Quantitative analysis of the average number of visible liver metastases. $n=6$ per group. (C) Kaplan-Meier survival curves for the mice in both groups in the mouse model of metastasis. (D) Changes in body weight in the mice in the mouse model of metastasis. " $\mathrm{P}<0.05$. PC, pancreatic cancer.

PC predicted less favorable outcomes (Fig. 1B; P<0.05). To verify the results obtained from the bioinformatics analysis, RT-qPCR was performed on the 54 pairs of PC tissues and adjacent matching tissues, and the results revealed that the expression of Linc00261 was also decreased in our PC tissues compared with adjacent non-tumor tissues (Fig. $1 \mathrm{C} ; \mathrm{P}<0.05$ ). A $\chi^{2}$ test was performed to analyze the relationship between the expression of Linc00261 and various clinicopathological characteristics. The results revealed that increased expression of Linc00261 was significantly negatively associated with several clinical traits of PC, such as tumor size, lymph node metastasis, TNM stage, distant metastasis, perineural invasion and blood vessel invasion (Table I). Furthermore, it was demonstrated that the expression of Linc00261 was also reduced in all the PC cell lines compared with the control pancreatic epithelial cell line (Fig. 1D; P<0.05). Collectively, Linc00261 may serve as a tumor-suppressive lncRNA in PC.

Linc00261 overexpression inhibits cell migration and invasion, and EMT in PC cells. Then, PANC-1 and MIA-PaCa2 cell lines which were derived from primary tumors with different metastatic ability were employed for our study. The migratory ability of PC cells in MIA-PaCa 2 and PANC-1 cells transfected with empty vector or Linc00261 overexpression lentivirus was assessed using a wound healing assay. Linc00261 overexpression both significantly reduced migration of PANC-1 and MIA-PaCa2 cells (Fig. 2A; $\mathrm{P}<0.05)$. Similarly, Transwell invasion assays revealed that overexpression of Linc00261 both significantly suppressed the invasive capacity of PANC-1 and MIA-PaCa2 cells (Fig. 2B; $\mathrm{P}<0.05$ ). Various studies have demonstrated that epithelial-mesenchymal transition promotes the process of metastasis of cancer cells (22). The expression of several EMT markers, including E-cadherin, $\mathrm{N}$-cadherin and vimentin were assessed in MIA-PaCa2 and PANC-1 cells transfected with empty vector and Linc00261 overexpression lentivirus, and the results revealed that the expression of E-cadherin was increased in the Linc00261 overexpression cells, whereas the expression of $\mathrm{N}$-cadherin and vimentin were markedly reduced (Fig. 2C; $\mathrm{P}<0.05$ ). 

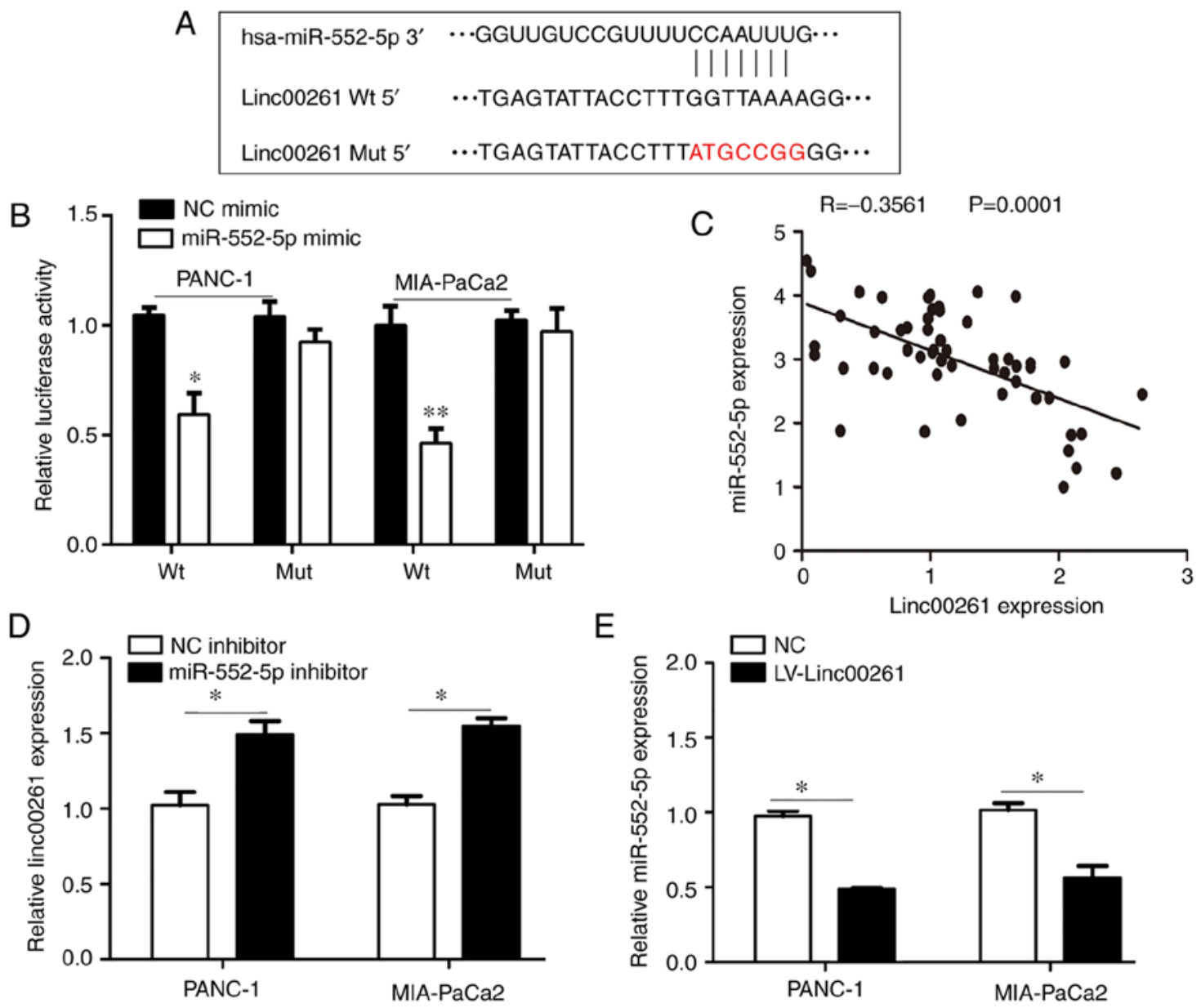

$\mathrm{E}$
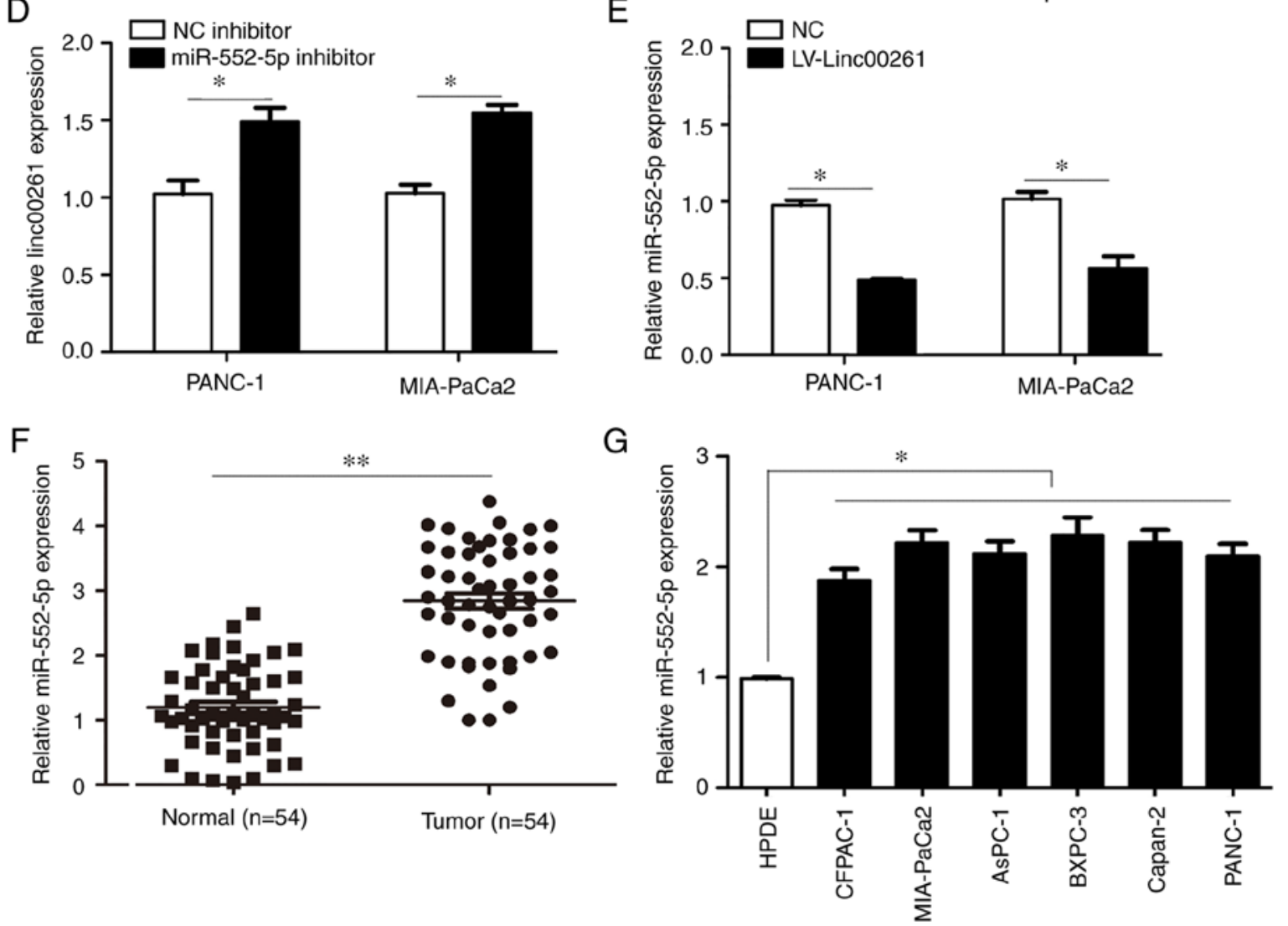

Figure 5. miR-552-5p is a target of Linc00261 in PC. (A) Linc00261 predicted binding site in the 3'UTR of miR-552-5p mRNA. (B) miR-552-5p mimics reduced luciferase activity in PC cells, this was not observed with the NC mimics. (C) Expression of Linc00261 was inversely associated with expression of miR-552-5p in PC tissues. (D) miR-552-5p inhibition increased the mRNA expression levels of Linc00261 in PC cells. (E) Overexpression of Linc00261 decreases the expression of miR-552-5p. (F) The mRNA expression levels of miR-552-5p were detected in PC tissues and adjacent non-tumor tissues using reverse transcription-quantitative PCR. (G) mRNA expression levels of miR-552-5p in the CFPAC-1, AsPC-1, MIA-PaCa2, Capan-2, BXPC-3 and PANC-1 PC cells were significantly higher compared with HPDE cells. ${ }^{*} \mathrm{P}<0.05,{ }^{* * *} \mathrm{P}<0.01$. PC, pancreatic cancer; UTR, untranslated region; NC, negative control; miR, microRNA; HPDE, human pancreatic epithelium cells.

Linc00261 knockdown increases migration and invasion, and EMT of PC cells. The results of the wound healing assays revealed that knockdown of Linc00261 significantly increased the migration of PANC-1 and MIA-PaCa2 cells (Fig. 3A; $\mathrm{P}<0.05)$. Similarly, the results of the Transwell invasion assays revealed that Linc00261 knockdown significantly increased invasion of PANC-1 and MIA-PaCa2 cells (Fig. 3B; P<0.05).
The results of western blotting revealed that Linc00261 knockdown significantly decreased the expression of E-cadherin, whereas the expression levels of $\mathrm{N}$-cadherin and vimentin were increased (Fig. 3C; $\mathrm{P}<0.05$ ).

Linc00261 reduces metastasis of PC cells in vivo. PANC-1 cells transfected with empty vector or Linc00261 overexpression lenti- 
A

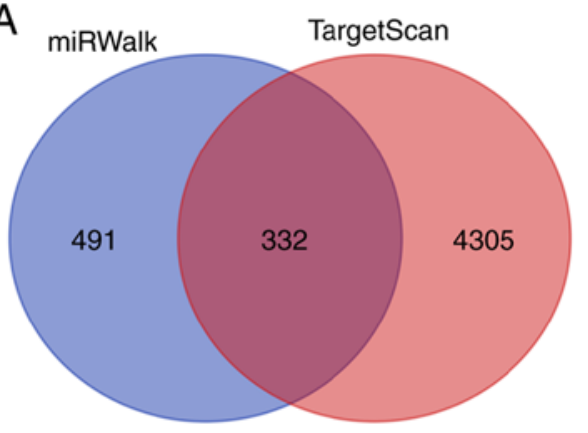

B Hsa05220:Chronic myeloid leukemia $\begin{aligned} & \text { Hsa05032:Morphine addiction } \\ & \text { Hsa0417:Prolactin signaling pathway }\end{aligned}$
Hsa04068:FoxO signaling pathway.
Hsa04071:Sphingolipid signaling pathway $\begin{aligned} & \text { Hsa05032:Morphine addiction } \\ & \text { Hsa0417:Prolactin signaling pathway }\end{aligned}$
Hsa04068:FoxO signaling pathway.
Hsa04071:Sphingolipid signaling pathway $\begin{aligned} & \text { Hsa05032:Morphine addiction } \\ & \text { Hsa0417:Prolactin signaling pathway }\end{aligned}$
Hsa04068:FoxO signaling pathway.
Hsa04071:Sphingolipid signaling pathway

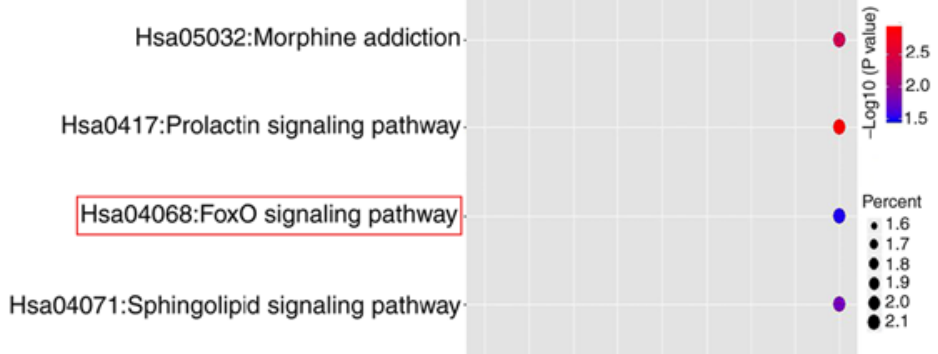

Term 7.0
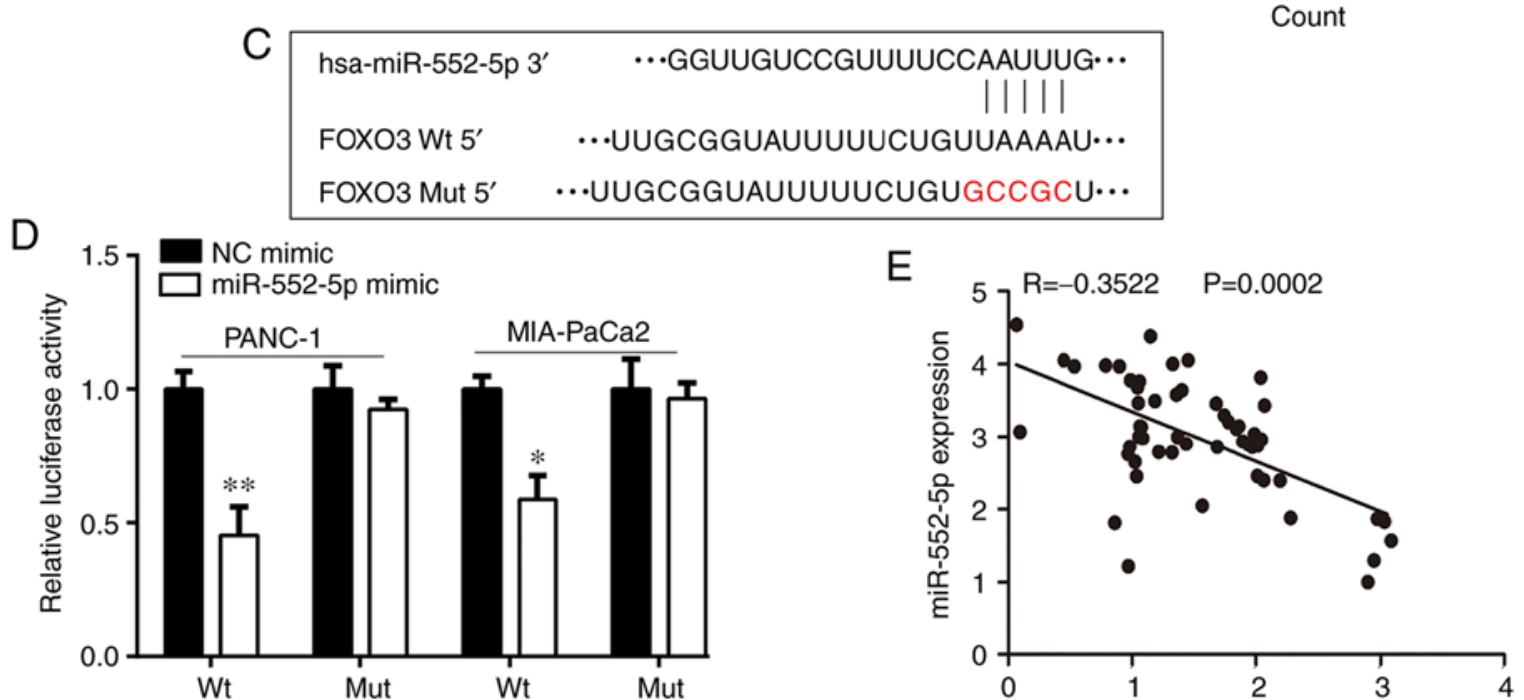

E
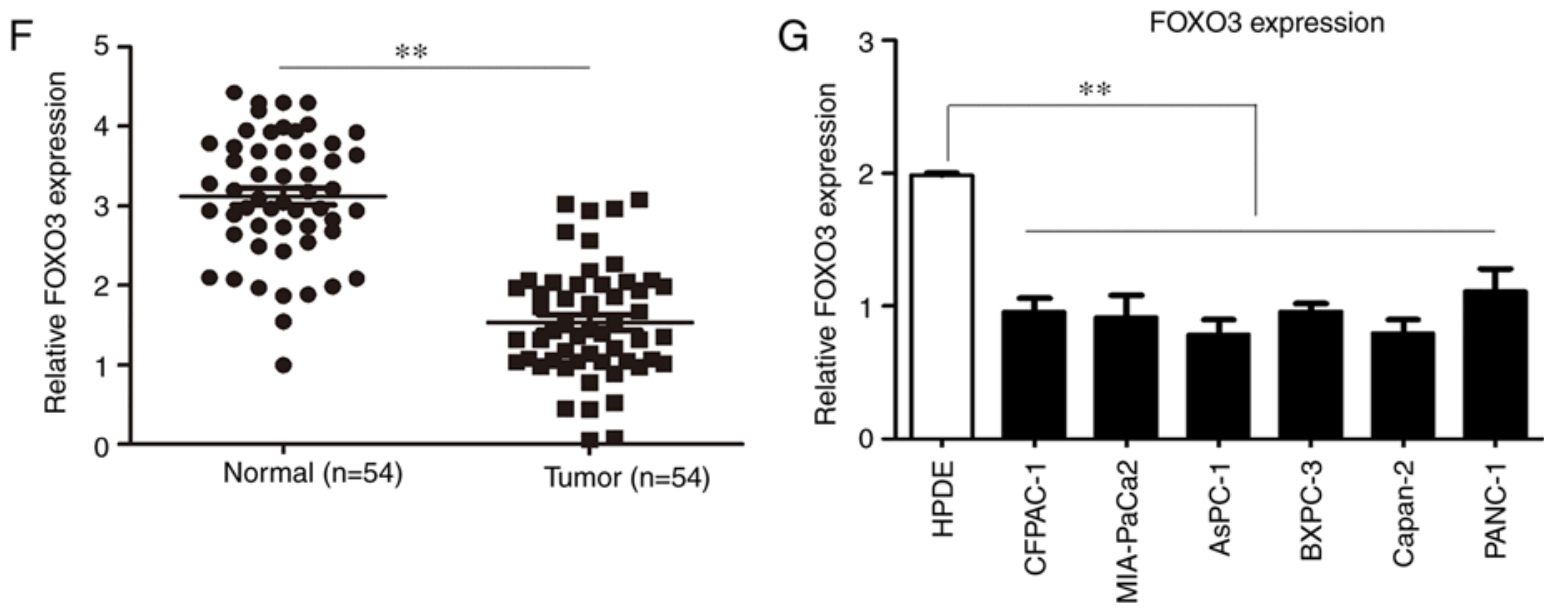

Figure 6. FOXO3 is a target of miR-552-5p in PC. (A) Using TargetScan and miRwalk online databases, the target genes of miR-552-5p were predicted and 332 genes were considered relevant based on the results from both databases. (B) Bubble chart showing the pathways in which miR-552-5p targeted genes are enriched in. (C) miR-552-5p was predicted to bind to the 3'UTR of FOXO3 mRNA. The mutated site is highlighted. (D) miR-552-5p mimics reduced the luciferase intensity in PC cells, whereas mutation of the associated element in 3'-UTR of FOXO3 mRNA abolished the effect of miR-552-5p mimic on luciferase activity. (E) The expression of miR-552-5p was inversely associated with expression of FOXO3 in PC tissues. (F) The mRNA expression levels of FOXO3 in PC tissues and adjacent non-tumor tissues. (G) The mRNA expression levels of FOXO3 in CFPAC-1, AsPC-1, MIA-Paca2, Capan-2, BXPC-3 and PANC-1 PC cells were significantly decreased compared with HDPE cells. ${ }^{*} \mathrm{P}<0.05,{ }^{* *} \mathrm{P}<0.01$. PC, pancreatic cancer; miR, microRNA; UTR, untranslated region; HPDE, human pancreatic epithelium cells; FOXO3, forkhead box O3.

virus were injected into the spleen of nude mice. After 10 weeks, metastasis of PANC-1 cells was assessed, and the results revealed that the number of metastatic foci was significantly decreased in the mice injected with the Linc00261 cells (Fig. 4A and B; $\mathrm{P}<0.05$ ). Similarly, the rate of death in mice was significantly lower in the mice injected with Linc00261-overexpressing cells compared with the mice injected with the empty vector control cells (Fig. 4C; $\mathrm{P}<0.05$ ), as well as weight loss (Fig. 4D, $\mathrm{P}<0.05$ ). Collectively, these results indicated that Linc00261 suppressed metastasis of PC cells in vivo. 
A

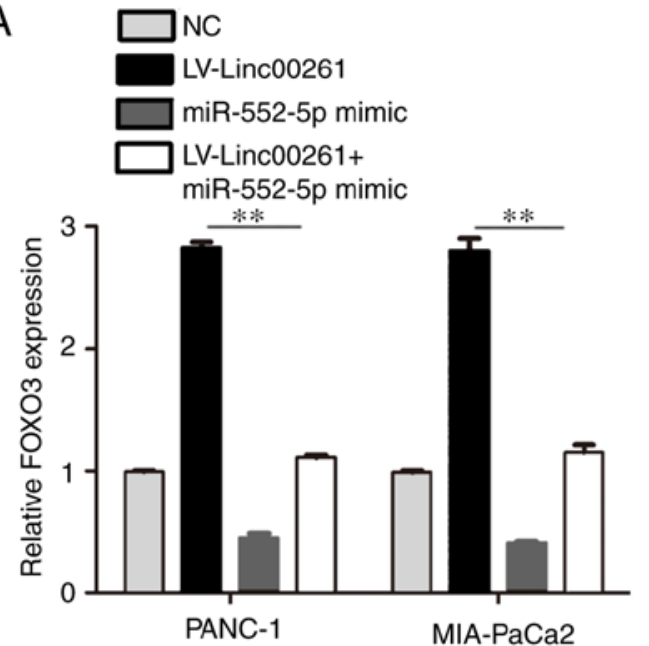

C
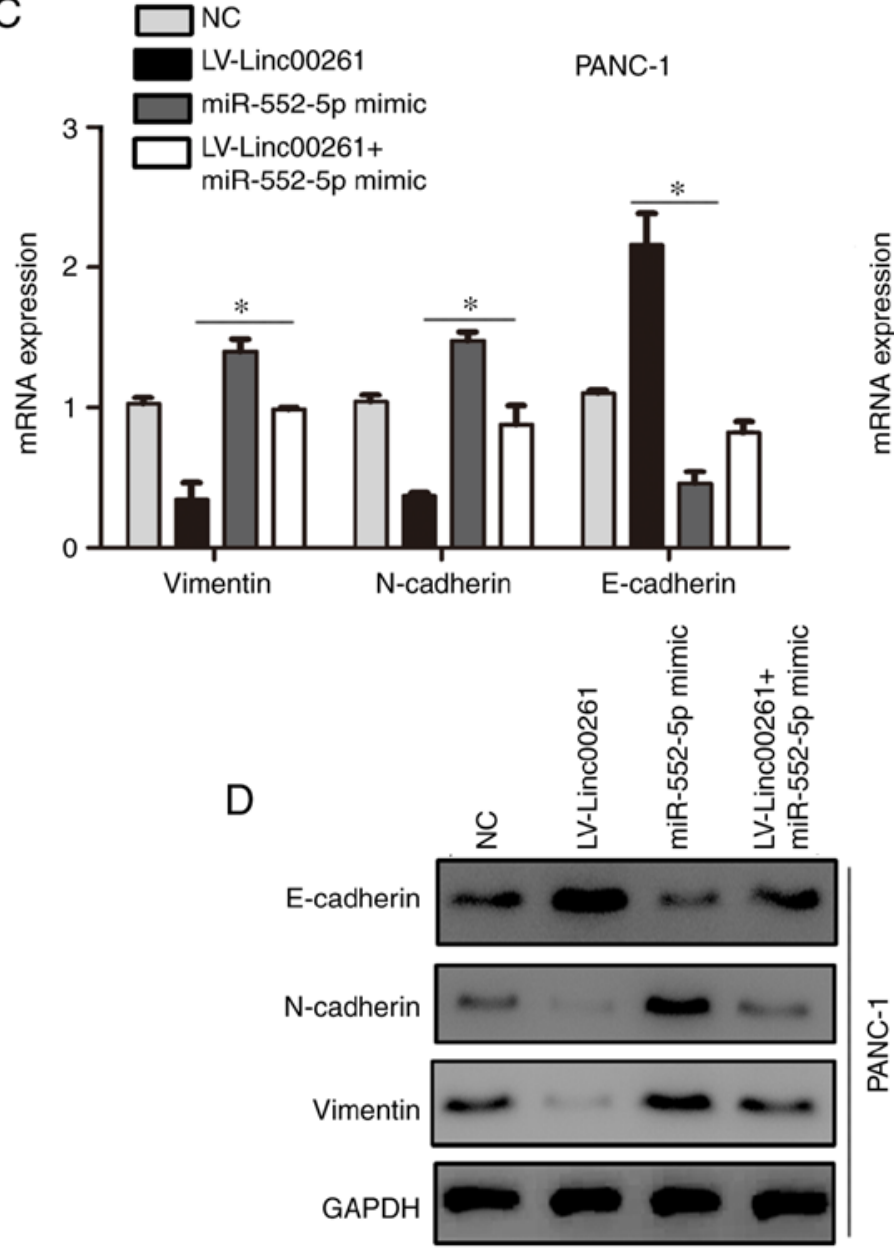

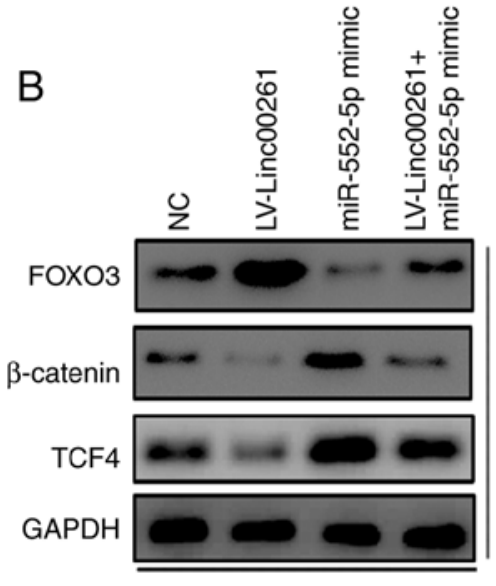

PANC-1

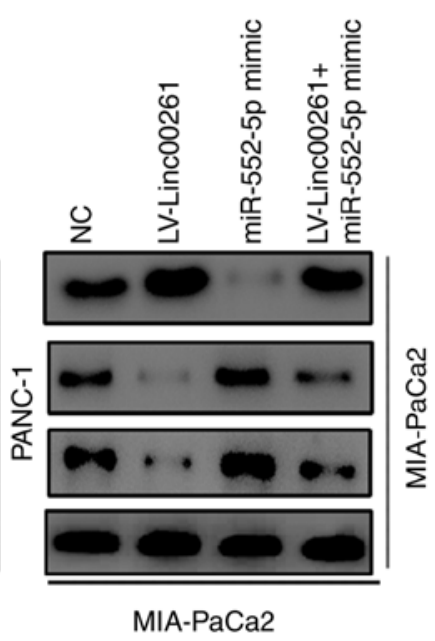

MIA-PaCa2
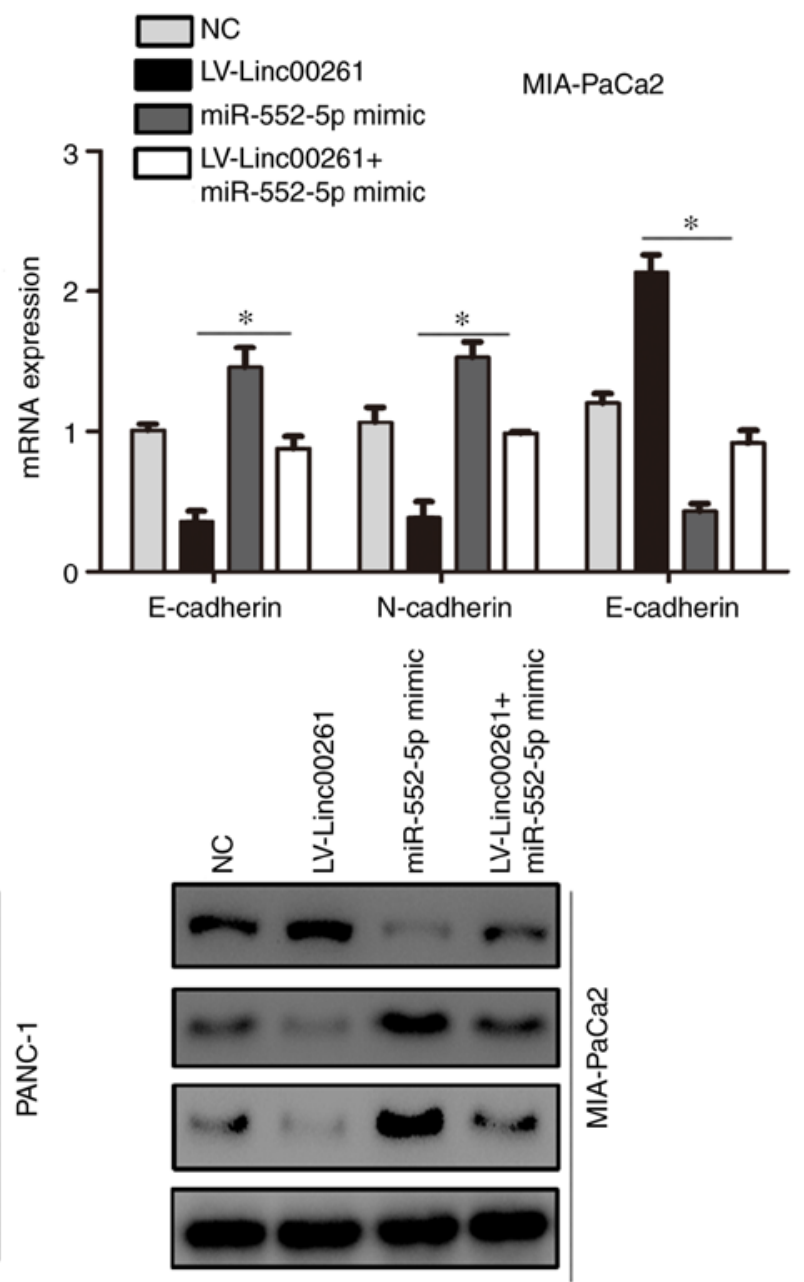

Figure 7. Linc00261 regulates the $\beta$-catenin/TCF4 signaling pathway via miR-552-5p/FOXO3. Cells were divided into four groups: NC lentivirus; LV-Linc00261; miR-552-5p mimics; and LV-Linc00261+miR-552-5p mimics. (A) The mRNA expression levels of FOXO3 in each group were detected using reverse transcription-quantitative PCR. (B) The protein expression levels of FOXO3, $\beta$-catenin and TCF4 in each group were detected using western blotting. (C) The mRNA expression levels of E-cadherin, $\mathrm{N}$-cadherin and vimentin in each group. (D) The protein expression levels of E-cadherin, $\mathrm{N}$-cadherin and vimentin in each group. ${ }^{*} \mathrm{P}<0.05,{ }^{* *} \mathrm{P}<0.01$. miR, microRNA; FOXO3, forkhead box O3; NC, negative control; LV-Linc00261, Linc00261 overexpression lentivirus; miR, microRNA.

miR-552-5p is a target of Linc00261 in PC. Target miRNAs of Linc00261 were predicted using TargetScan, and the analysis revealed that miR-552-5p possessed potential binding sites for Linc00261 (Fig. 5A). A dual luciferase reporter assay was performed and the results revealed that luciferase activity was decreased in PANC-1 and MIA-PaCa2 cells co-transfected with miR-552-5p mimic and Linc00261-WT plasmid compared with cells co-transfected with NC mimic and the Linc00261-WT plasmid (Fig. 5B; $\mathrm{P}<0.05$ ). The expression levels of miR-552-5p were inversely associated with Linc00261 (Fig. 5C; r=-0.3561; 


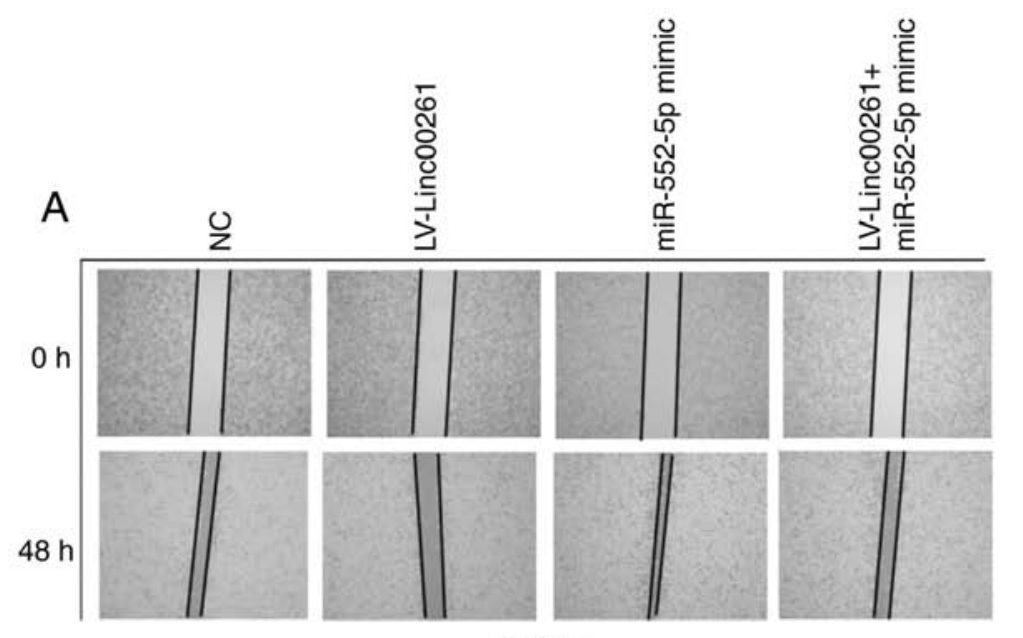

PANC-1
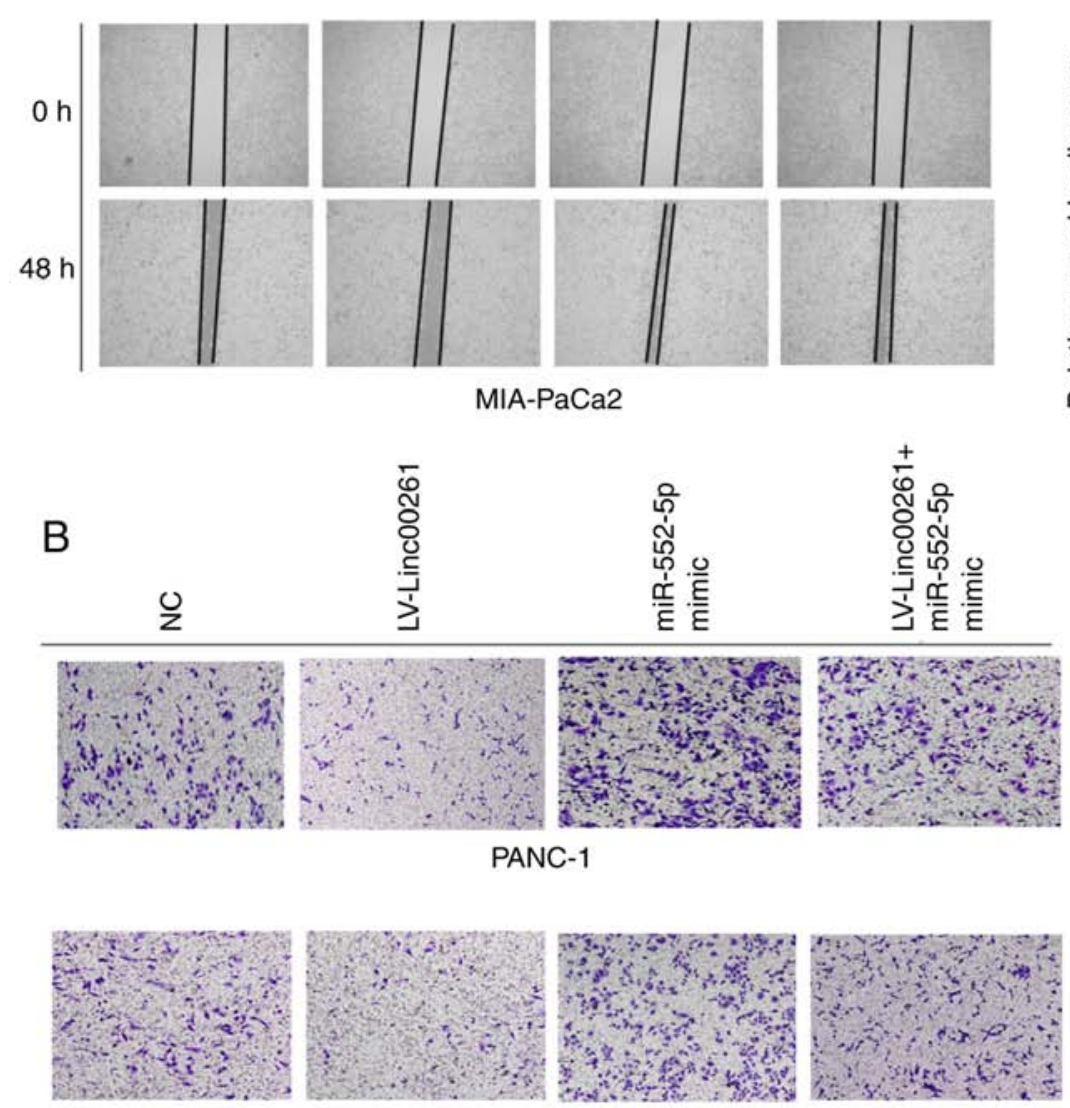

MIA-PaCa2
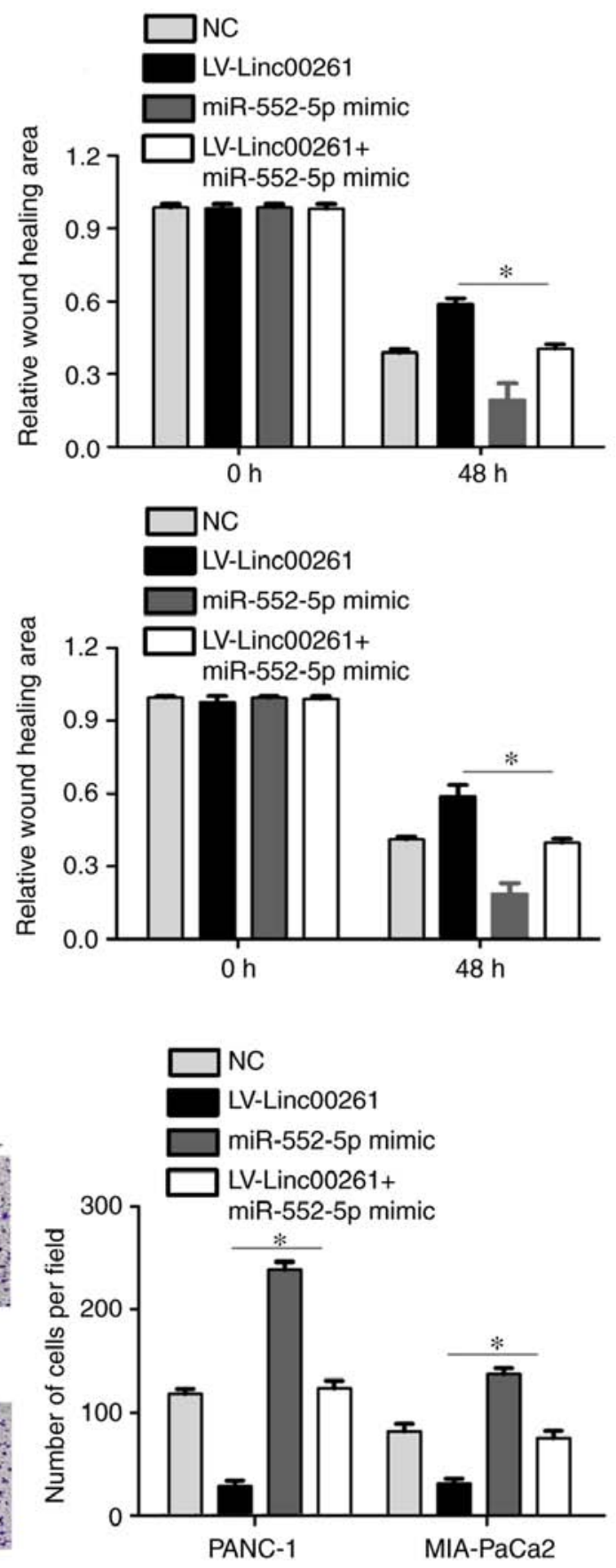

Figure 8. miR-552-5p overexpression reverses the inhibitory effects of Linc00261. Cells were divided into four groups: NC lentivirus; LV-Linc00261; miR-552-5p mimics; and LV-Linc00261+miR-552-5p mimics. (A) Wound healing assays were used to assess the migratory ability of each group. (B) Transwell invasion assays were used to assess the invasive ability of each group. ${ }^{*} \mathrm{P}<0.05$. miR, microRNA; NC, negative control; LV-Linc00261, Linc00261 overexpression lentivirus.

$\mathrm{P}<0.01)$. Transfection of miR-552-5p inhibitor into PANC-1 and MIA-PaCa2 cells increased the expression of Linc00261 (Fig. 5D; P<0.05). Similarly, the expression levels of miR-552-5p were significantly decreased in Linc00261-overexpressed cells (Fig. 5E; $\mathrm{P}<0.05$ ). In addition, the expression of miR-552-5p was increased in PC tissues and PC cell lines (Fig. 5F and G; $\mathrm{P}<0.05)$. These results indicated that miR-552-5p may be an important target of Linc00261 in PC.
miR-552-5p upregulates the expression of FOXO3 in PC. Since miR-552-5p was considered an important target of Linc00261 in PC, the targets of miR-552-5p were predicted. Using the online tools, miRWalk and TargetScan, a total of 332 potential target genes regulated by miR-552-5p were predicted (Fig. 6A). These target genes were primarily enriched for 5 pathways, including the FOXO signaling pathway (Fig. 6B). FOXO3, which is involved in the FOXO signaling pathway was 
determined to possess binding sites for miR-552-5p (Fig. 6C). Due to evidence that FOXO3 serves a key role in PC (20), our focus was on FOXO3. Luciferase activity was significantly reduced in cells co-transfected with miR-552-5p mimic and FOXO3-WT plasmid compared with cells co-transfected with miR-552-5p mimic and FOXO3-Mut plasmid (Fig. 6D; $\mathrm{P}<0.05)$. Furthermore, the expression of FOXO3 was decreased in tumor samples, and negatively associated with the expression of miR-552-5p in PC (Fig. 6E and F; P<0.05). Similarly, the mRNA levels of FOXO3 in PC cell lines were also decreased compared with the normal pancreatic epithelial cell line (Fig. 6G; $\mathrm{P}<0.05$ ). Collectively, FOXO3 was considered to be an important target of miR-552-5p in PC.

Linc00261 inhibits the activity of the $\beta$-catenin/TCF4 pathway and cell metastasis via a miR-552-5p/FOXO3 axis. To determine whether the effect of Linc00261 on PC was dependent on miR-552-5p/FOXO3, the expression of miR-552-5p was restored in Linc00261-overexpressing cells. miR-552-5p overexpression increased the mRNA and protein expression levels of FOXO3 (Fig. 7A and B; P<0.05). Since it has been demonstrated that FOXO3 regulates the Wnt signaling pathway (23), the expression of $\beta$-catenin and TCF4 was assessed. The results revealed that Linc00261 overexpression decreased the expression of $\beta$-catenin and TCF4, whereas miR-552-5p prevented the inhibitory effects of Linc00261 on the Wnt signaling pathway (Fig. 7B; $\mathrm{P}<0.05)$. The expression levels of E-cadherin, $\mathrm{N}$-cadherin and vimentin, which are also downstream proteins of TCF4, were assessed using RT-qPCR and western blotting. Restoration of miR-552-5p alleviated the effects of Linc00261 overexpression on the expression of E-cadherin, N-cadherin and vimentin (Fig. 7C and D; $\mathrm{P}<0.05)$. Similarly, miR-552-5p reversed the inhibitory effects of Linc00261 on the migration and invasion of PC cells (Fig. 8A and $\mathrm{B} ; \mathrm{P}<0.05)$.

\section{Discussion}

PC exhibits a high metastatic capacity, thus any factor which promotes metastasis of PC may be involved in the progression of PC. LncRNAs have been revealed to regulate the metastasis of PC previously (24). Since Linc00261 has been demonstrated to function as a tumor-suppressive LincRNA in a range of different types of cancer $(25,26)$, it was hypothesized that Linc00261 may also participate in the pathogenesis of PC.

Consistent with previous studies, in the present study, Linc00261 expression was decreased in PC tissues and 6 PC cell lines compared with the adjacent non-tumor tissues and normal pancreatic epithelial cells, respectively. Reduced expression of Linc00261 predicted less favorable outcomes in patients with PC and Linc00261 overexpression decreased PC cell migration and invasion in vitro as well as EMT, whereas Linc00261 knockdown increased cell migration and invasion in vitro. Similarly, Linc00261 overexpression decreased PC cell metastasis in vivo. Together these data indicated that Linc00261 is a potential tumor-suppressive lncRNA in PC. Therefore, determining the underlying mechanism by which Linc00261 reduced metastasis of PC cells may assist in understanding the development of PC.
Numerous studies have demonstrated that specific lncRNAs may act as ceRNAs which are primarily located in the cytosol $(27,28)$. In the present study, through bioinformatics analysis and dual luciferase activity assays, Linc00261 was revealed to bind with miR-552-5p in PC cells. A previous study revealed that miR-552-5p acted as an oncogenic miRNA in osteosarcoma and promoted the development of osteosarcoma (29). Therefore, Linc00261 may inhibit PC cell metastasis by sponging miR-552-5p. The results of the present study revealed that the expression of miR-552-5p was negatively associated with Linc00261 in PC tissues. Linc00261 overexpression significantly decreased the expression of miR-552-5p in PC cells. Furthermore, the expression of miR-552-5p was significantly upregulated in PC tissues and cell lines. Therefore, miR-552-5p was considered a target miRNA of Linc00261 in PC.

Using bioinformatics analysis to predict the target genes of miR-552-5p, it was demonstrated that the target genes were significantly enriched in the FOXO signaling pathway and FOXO3 was a direct target of miR-552-5p. Since the FOXO pathway, and specifically FOXO3 can regulate the Wnt signaling pathway, which is involved in the development of PC (18), a focus was placed on FOXO3 and it was revealed that miR-552-5p overexpression significantly decreased its expression. Additionally, the expression of FOXO3 was negatively associated with miR-552-5p. Linc00261 overexpression significantly decreased the expression of FOXO3, and inhibited the Wnt signaling pathway and thus EMT. Ectopically restoring miR-552-5p expression in Linc00261-overexpressing cells significantly alleviated the inhibitory effect of Linc00261 on the expression of FOXO3, the Wnt pathway and EMT. Similarly, Linc00261 significantly decreased the migratory and invasive capacities of PC cells, whereas miR-552-5p restoration in Linc00261-overexpressing PC cells significantly increased the migration and invasion of PC cells.

In conclusion, the present study demonstrated that Linc00261 is a tumor-suppressive lncRNA in PC. Linc00261 inhibited PC cell metastasis in vitro and in vivo by regulating the miR-552-5p/FOXO3 axis. These results may assist in improving our understanding of the molecular mechanisms underlying the development and metastasis of PC, and indicates that Linc00261 may be a potential biomarker for diagnosis and target for treatment.

\section{Acknowledgements}

Not applicable.

\section{Funding}

The present study was supported by the National Natural Science Foundation of China (grant no. 81060176) and Major Projects of Applied and Basic Research Program of Guizhou Province (grant no. J-[2015]2003).

\section{Availability of data and materials}

The datasets used and/or analyzed during the present study are available from the corresponding author on reasonable request. 


\section{Authors' contributions}

TC, SL and ZZ collected and interpreted the data. TC, SL, ZZ, JZ, YX, YS, JL, SX, DM, BG analyzed the data. TC wrote the manuscript. All authors have read and approved the final version of the article.

\section{Ethics approval and consent to participate}

All patients who had provided samples provided informed consent. The present study was approved by the Ethics Committee of the Guizhou Medicine University (Approval no. 2019LS146) and was performed in accordance with the Declaration of Helsinki. All animal experiments were approved by the Ethics Committee of Guizhou Medical University (Approval no. 1900069).

\section{Patient consent for publication}

Not applicable.

\section{Competing interests}

The authors declare that they have no competing interests.

\section{References}

1. Lee SH, Chang PH, Chen PT, Lu CH, Hung YS, Tsang NM, Hung CY, Chen JS, Hsu HC, Chen YY and Chou WC: Association of time interval between cancer diagnosis and initiation of palliative chemotherapy with overall survival in patients with unresectable pancreatic cancer. Cancer Med 8: 3471-3478, 2019.

2. Skau Rasmussen L, Vittrup B, Ladekarl M, Pfeiffer P, Karen Yilmaz M, Østergaard Poulsen L, Østerlind K, Palnæs Hansen C, Bau Mortensen M, Viborg Mortensen F, et al: The effect of postoperative gemcitabine on overall survival in patients with resected pancreatic cancer: A nationwide population-based Danish register study. Acta Oncol 58: 864-871, 2019.

3. Elaileh A, Saharia A, Potter L, Baio F, Ghafel A, Abdelrahim M and Heyne K: Promising new treatments for pancreatic cancer in the era of targeted and immune therapies. Am J Cancer Res 9: 1871-1888, 2019

4. Mitschke J, Burk UC and Reinheckel T: The role of proteases in epithelial-to-mesenchymal cell transitions in cancer. Cancer Metastasis Rev 38: 431-444, 2019.

5. Hu X, Zhai Y, Kong P, Cui H, Yan T, Yang J, Qian Y, Ma Y, Wang F, Li H, et al: FAT1 prevents epithelial mesenchymal transition (EMT) via MAPK/ERK signaling pathway in esophageal squamous cell cancer. Cancer Lett 397: 83-93, 2017.

6. Baek SH, Ko JH, Lee JH, Kim C, Lee H, Nam D, Lee J, Lee SG, Yang WM, Um JY, et al: Ginkgolic acid inhibits invasion and migration and TGF- $\beta$-induced EMT of lung cancer cells through PI3K/Akt/mTOR inactivation. J Cell Physiol 232: 346-354, 2017.

7. Yang Y, Bai YS and Wang Q: CDGSH Iron Sulfur domain 2 activates proliferation and EMT of pancreatic cancer cells via $\mathrm{Wnt} / \beta$-catenin pathway and has prognostic value in human pancreatic cancer. Oncol Res 25: 605-615, 2017.

8. Kleszcz R: The canonical Wnt pathway. Postepy Biochem 65: 183-192, 2019 (In Polish).

9. Lin Y, Dong C and Zhou BP: Epigenetic regulation of EMT: The Snail story. Curr Pharm Des 20: 1698-1705, 2014.

10. Bhan A, Soleimani M and Mandal SS: Long noncoding RNA and cancer: A new paradigm. Cancer Res 77: 3965-3981, 2017.
11. Huang X, Zhi X, Gao Y, Ta N, Jiang H and Zheng J: LncRNAs in pancreatic cancer. Oncotarget 7: 57379-57390, 2016.

12. Yang J, Ye Z, Mei D, Gu H and Zhang J: Long noncoding RNA DLX6-AS1 promotes tumorigenesis by modulating miR-497-5p/FZD4/FZD6/Wnt/ $\beta$-catenin pathway in pancreatic cancer. Cancer Manag Res 11: 4209-4221, 2019.

13. Sun Y, Zhu Q, Yang W, Shan Y, Yu Z, Zhang Q and Wu H: LncRNA H19/miR-194/PFTK1 axis modulates the cell proliferation and migration of pancreatic cancer. J Cell Biochem 120: 3874-3886, 2019.

14. Wang ZK, Yang L, Wu LL, Mao H, Zhou YH, Zhang PF and Dai GH: Long non-coding RNA LINC00261 sensitizes human colon cancer cells to cisplatin therapy. Braz J Med Biol Res 51: e6793, 2017.

15. Yu Y, Li L, Zheng Z, Chen S, Chen E and Hu Y: Long non-coding RNA linc00261 suppresses gastric cancer progression via promoting Slug degradation. J Cell Mol Med 21: 955-967, 2017.

16. Shi J, Ma H, Wang H, Zhu W, Jiang S, Dou R and Yan B: Overexpression of LINC00261 inhibits non-small cell lung cancer cells progression by interacting with miR-522-3p and suppressing Wnt signaling. J Cell Biochem 120: 18378-18387, 2019.

17. Yan D, Liu W, Liu Y and Luo M: LINC00261 suppresses human colon cancer progression via sponging miR-324-3p and inactivating the Wnt $/ \beta$-catenin pathway. J Cell Physiol 234: 22648-22656, 2019.

18. Liu H, Yin J, Wang H, Jiang G, Deng M, Zhang G, Bu X, Cai S, Du J and He Z: FOXO3a modulates WNT/ $\beta$-catenin signaling and suppresses epithelial-to-mesenchymal transition in prostate cancer cells. Cell Signal 27: 510-518, 2015.

19. Yao S, Fan LY and Lam EW: The FOXO3-FOXM1 axis: A key cancer drug target and a modulator of cancer drug resistance. Semin Cancer Biol 50: 77-89, 2018.

20. Li J, Yang R, Dong Y, Chen M, Wang Y and Wang G: Knockdown of FOXO3a induces epithelial-mesenchymal transition and promotes metastasis of pancreatic ductal adenocarcinoma by activation of the $\beta$-catenin/TCF4 pathway through SPRY2.J Exp Clin Cancer Res 38: 38, 2019.

21. Livak KJ and Schmittgen TD: Analysis of relative gene expression data using real-time quantitative PCR and the 2(-Delta Delta C(T)) method. Methods 25: 402-408, 2001.

22. Chaffer CL, San Juan BP, Lim E and Weinberg RA: EMT, cell plasticity and metastasis. Cancer Metastasis Rev 35: 645-654, 2016.

23. Iyer S, Ambrogini E, Bartell SM, Han L, Roberson PK, de Cabo R, Jilka RL, Weinstein RS, O'Brien CA, Manolagas SC and Almeida M: FOXOs attenuate bone formation by suppressing Wnt signaling. J Clin Invest 123: 3409-3419, 2013.

24. Previdi MC, Carotenuto P, Zito D, Pandolfo R and Braconi C: Noncoding RNAs as novel biomarkers in pancreatic cancer: What do we know? Future Oncol 13: 443-453, 2017.

25. Wang H, Sha L, Huang L, Yang S, Zhou Q, Luo X and Shi B: LINC00261 functions as a competing endogenous RNA to regulate BCL2L11 expression by sponging miR-132-3p in endometriosis. Am J Transl Res 11: 2269-2279, 2019.

26. Cheng D, Jiang S, Chen J, Li J, Ao L and Zhang Y: Upregulated long noncoding RNA Linc00261 in pre-eclampsia and its effect on trophoblast invasion and migration via regulating miR-558/TIMP4 signaling pathway. J Cell Biochem 120: 13243-13253, 2019

27. Lin K, Jiang H, Zhuang SS, Qin YS, Qiu GD, She YQ, Zheng JT, Chen C, Fang L and Zhang SY: Long noncoding RNA LINC00261 induces chemosensitization to 5-fluorouracil by mediating methylation-dependent repression of DPYD in human esophageal cancer. FASEB J 33: 1972-1988, 2019.

28. Fang Q, Sang L and Du S: Long noncoding RNA LINC00261 regulates endometrial carcinoma progression by modulating miRNA/FOXO1 expression. Cell Biochem Funct 36: 323-330, 2018.

29. Cai W, Xu Y, Yin J, Zuo W and Su Z: miR-552-5p facilitates osteosarcoma cell proliferation and metastasis by targeting WIF1. Exp Ther Med 17: 3781-3788, 2019.

This work is licensed under a Creative Commons Attribution-NonCommercial-NoDerivatives 4.0 International (CC BY-NC-ND 4.0) License. 